\title{
1 A viral histone-like protein exploits antagonism between linker histones and HMGB 2 proteins to obstruct the cell cycle

4 Kelsey L. Lynch ${ }^{1}$, Mongoljin Bat-Erdene ${ }^{1}$, Melanie R. Dillon ${ }^{1}$, Hannah C. Lewis ${ }^{1,2}$, Daphne C. 5 Avgousti ${ }^{1,2^{*}}$

${ }^{1}$ Human Biology Division, Fred Hutchinson Cancer Research Center, Seattle, WA, USA

$8{ }^{2}$ Molecular \& Cellular Biology in Seattle, Graduate Program, University of Washington and Fred

9 Hutchinson Cancer Research Center, Seattle, WA, USA

10 *address correspondence to avgousti@fredhutch.org

12 Virus infection necessarily requires redirecting cellular resources towards viral progeny 13 production. Adenovirus encodes the histone-like protein VII that causes catastrophic global 14 reorganization of host chromatin to promote virus infection. Protein VII recruits the family of high 15 mobility group box (HMGB) proteins to chromatin along with the histone chaperone SET. As a consequence of this recruitment, we find that protein VII causes chromatin-depletion of several linker histone $\mathrm{H} 1$ isoforms. The relationship between linker histone $\mathrm{H} 1$ and the functionally opposite HMGB proteins is critical for higher order chromatin structure. However, the physiological consequences of perturbing this relationship are largely unknown. Here, we employ complementary systems in Saccharomyces cerevisiae and human cells to demonstrate

21 that adenovirus protein VII disrupts the H1-HMGB balance to obstruct the cell cycle. We find

22 that protein VII causes an accumulation of G2/M cells both in yeast and human systems, 23 underscoring the high conservation of this chromatin vulnerability. In contrast, adenovirus E1A 24 and $\mathrm{E} 1 \mathrm{~B}$ proteins are well-established to override cell cycle regulation and promote 25 transformation of human cells. Strikingly, we find that protein VII obstructs the cell cycle even in 26 the presence of E1A and E1B, suggesting that protein VII-directed cell cycle disruption ensures 27 host resources are directed towards viral proliferation during infection. Together, our results 28 demonstrate that protein VII targets H1-HMGB1 antagonism to obstruct cell cycle progression, revealing an unexpected chromatin vulnerability exploited for viral benefit. 


\section{Introduction}

34 Viral takeover of cellular processes is essential for the success of these intracellular pathogens.

35 Controlling host chromatin is an integral aspect of viral infection that includes re-directing host

36 resources for transcription, DNA replication, and cell division towards viral replication. Several

37 examples have recently come to light that highlight viral alteration to host gene expression

38 through modifications of core histone tails ${ }^{1-7}$. Host chromatin may also be manipulated through

39 displacement of linker histones. Linker histones are critical for genome compaction ${ }^{8-10}$ and

40 compete for binding sites with high mobility group (HMG) proteins ${ }^{11}$, which in contrast promote

41 decompaction of chromatin and increase DNA accessibility ${ }^{12-15}$. This interplay between linker

42 histones and HMG proteins is a vulnerability that may be a target of viral manipulation.

44 The adenovirus genome is packaged with a small basic protein known as protein $\mathrm{VII}^{16-18}$. This

$4520 \mathrm{kDa}$ core protein forms a 'beads on a string' structure within virus particles and recruits the 46 histone chaperone protein SET ${ }^{19-21}$ onto viral genomes as they enter the nucleus. SET is 47 thought to populate the viral genomes with histones to promote viral gene expression ${ }^{22-25}$. The

48 first viral gene products, E1A and E1B, are then expressed leading to subsequent viral gene 49 activation, global changes in host histone modifications, and bypass of cell cycle checkpoints ${ }^{26-}$ 5028 . E1A has been the focus of many studies of cellular transformation and epigenetic 51 reprogramming by viruses ${ }^{29,30}$, although it remains unclear why transformation does not occur in 52 humans as a result of adenovirus infection ${ }^{26,31}$. Protein VII, along with other viral structural 53 proteins, is transcribed late during infection ${ }^{28}$. In addition to its structural role inside virion cores, 54 protein VII localizes to host chromatin and disrupts normal nuclear processes ${ }^{32,33}$. Protein VII is 55 post-translationally modified and these modifications regulate protein VII's localization to 56 chromatin ${ }^{32,33}$. Recombinant protein VII binds purified nucleosomes directly, protecting 57 approximately 165 bp of DNA from digestion by micrococcal nuclease, suggesting that it may 58 have similar binding sites as the linker histones ${ }^{32}$.

Several host proteins are significantly enriched in chromatin in the presence of protein VII as 61 measured by salt fractionation followed by mass spectrometry ${ }^{32,34}$, including $\mathrm{SET}^{35}$ and the 62 family of high mobility group box $(\mathrm{HMGB})^{14}$ proteins. Without protein VII, SET and HMGB 63 proteins are transiently bound to chromatin. SET, also known as TAF-IB, has been described as 64 a linker histone chaperone ${ }^{36,37}$ while HMGB1 has been shown to act as an antagonist of histone $65 \mathrm{H} 1^{12,13,15}$. Here, we use complementary systems in Saccharomyces cerevisiae and human cells 
66 to show that adenovirus protein VII requires HMGB1 and SET to disrupt chromatin and cause

67 cell growth defects. We determine that these deficiencies in growth are due to deviations in cell

68 cycle progression. We find that protein VII can slow cell cycle progression even in the presence

69 of adenovirus E1A, which normally leads to a bypass of cell cycle checkpoints thereby 70 promoting transformation. Therefore, we propose that during adenovirus infection protein VII

71 obstructs mitosis to ensure cellular resources are directed towards viral progeny production

72 rather than cell division. This study is the first to show viral exploitation of the H1-HMGB1

73 interaction and subsequent delay of the cell cycle for viral benefit.

\section{Results}

76 Human adenovirus protein VII causes growth defects in budding yeast

77 In addition to the enrichment of SET and HMGBs in chromatin upon protein VII expression in 78 human lung epithelial cells, we discovered that several isoforms of the linker histone $\mathrm{H} 1$ were 79 significantly depleted from chromatin in the presence of protein VII (Supplemental Figure 1a).

80 Depletion of linker histones has recently come to light as a regulatory mechanism for diverse 81 cellular outcomes ${ }^{38-40}$. Thus, our surprising finding led us to hypothesize that protein VII

82 harnesses the antagonism between HMGB1 and H1 by co-opting SET and HMGB1 to displace 83 histone $\mathrm{H} 1$ in host chromatin (Supplemental Figure 1b). We decided to test our model with 84 genetic studies in Saccharomyces cerevisiae, which faithfully represents many aspects of mammalian chromatin with less genetic redundancy than mammalian cells ${ }^{41,42}$.

We generated a selectable, inducible expression plasmid bearing a yeast codon-optimized version of mature protein VII from adenovirus type 5 (Supplemental Figure 1c). We introduced the inducible expression plasmid into the wild type (WT) lab strain W303 and assayed growth on solid media using a serial dilution growth assay (Figure 1a). Strikingly, we found that expression of protein VII in budding yeast resulted in a $\sim 40 \%$ growth deficit compared to cells expressing GFP (Figure 1b). Furthermore, upon mutation of five post-translationally modified (PTM) sites previously found to impact protein VIl's localization to chromatin ${ }^{32,33}$, the growth defect was rescued (Figure 1a and b). We further characterized the growth defect by inducing expression during log phase growth in liquid culture and calculating the exponential growth rate. Without the induction of expression, there was no difference in exponential growth rate among the three

97 strains (Figure 1c). However, expression of protein VII caused a significant reduction in growth 98 compared to GFP-expressing cells. The growth defect was rescued upon mutation of protein VII 99 and the exponential growth rate of cells expressing VII- $\triangle$ PTM was comparable to GFP. Since 
protein expression is induced by galactose, a less-preferred energy source, the absolute growth rates for all induced strains were lower than the same strains grown in dextrose media without transgene expression, as expected. We confirmed comparable expression of each protein during exponential growth by western blot (Figure 1d). Furthermore, we found that the growth defect caused by protein VII was not unique to the W303 strain as we observed comparable results in an alternate lab strain, FY602 (Figure1e-h). The similar outcomes in both strains highlight the robust and conserved nature of protein VII's impact on yeast.

We found by immunofluorescence microscopy that protein VII is localized to the yeast nucleus (Supplemental Figure 2a), likely as a result of the endogenous nuclear localization signal (NLS) at the $\mathrm{N}$-terminus of protein $\mathrm{VII}^{43}$. Therefore, we assessed whether protein $\mathrm{VII}$ bound the yeast genome directly by chromatin immunoprecipitation followed by sequencing (ChIP-seq) using an endogenous antibody against protein $\mathrm{VII}^{44}$ (Supplemental Figure 2b, Supplemental Figure 3). ChIP-seq showed that protein VII binds throughout the yeast genome. We identified local variability in protein VII binding compared to nucleosomes as determined by total histone $\mathrm{H} 3$ ChIP. To test for enrichment of protein VII, we used the MACS2 peak calling algorithm to compare protein VII localization both to the $\mathrm{H} 3$ control and to the input control ${ }^{45}$. MACS2 identified very few peaks of protein VII enrichment compared to the input. However, only 9 of those peaks were also called using $\mathrm{H} 3$ as the control condition which suggests that they are not bona fide sites of enrichment and that protein VII coats yeast chromatin uniformly just as histone H3 does. Thus, we conclude that protein VII directly binds chromatin throughout the genome.

121 Together these data demonstrate that the effects of protein VII on chromatin in budding yeast

122 are direct and robust, indicating that eukaryotic chromatin is widely vulnerable to protein VII.

124 The genetic interactions of protein VII with the yeast homologs of H1, HMGB1, and SET 125 support a linker histone displacement model

127 Unlike mammals, which have multiple linker $\mathrm{H} 1$ and $\mathrm{HMGB}$ proteins ${ }^{10,14}$, yeast have primary 128 homologs of linker histone H1, HMGB1 and SET, which are Hho1, Hmo1, and Nap1 129 respectively ${ }^{46-51}$. To test whether the budding yeast proteins Hho1, Hmo1, and Nap1 interact 130 genetically with protein VII, we introduced the protein VII expression plasmid into strains deleted 131 for each of these genes as well as a double mutant lacking both HMO1 and NAP1. We then 132 assessed whether the deletions affected the growth defect caused by protein VII. We 133 hypothesized that Hmo1 and Nap1, which correspond to the known binding partners HMGB1 
134 and SET respectively, facilitate protein VII dysregulation of chromatin (Figure 2a). Based on this 135 model, we expected that deletion of either the HMO1 or NAP1 gene would prevent protein VII 136 from disrupting growth and therefore rescue the growth defects caused by protein VII. Indeed, 137 we found that loss of HMO1 or NAP1 during protein VIl expression rescued growth compared to 138 WT (Figure 2b). Relative growth with protein VII was significantly increased in $h m o 1 \Delta$ compared 139 to WT in the W303 strain (Figure 2c). Likewise, deletion of NAP1 led to slightly better relative 140 growth compared to WT, though quantification did not reach significance. We also assayed 141 growth with protein VII expression in the same set of gene deletions in the alternate FY602 142 strain and found that deletion of either HMO1 or NAP1 significantly rescued growth (Figure 2e$143 \mathrm{f})$. These minor variations in the degree of rescue between the two different strains is likely due 144 to differences in genotype. Taken together, we conclude that both Hmo1 and Nap1 are 145 important for protein VII to cause reduced growth.

We hypothesized that Hmo1 and Nap1 function cooperatively to facilitate protein VII's disruption of chromatin. As such, removing both factors should have the same effect on the protein VII growth defect as deleting either gene independently. To test this, we assayed growth with protein VII expression in a $h m 01 \Delta$ nap $1 \Delta$ double deletion strain. Consistent with our hypothesis, we found that deletion of both genes rescued the growth defects caused by protein VII to the same degree as a single gene deletion (Figure 2b and e). For both W303 and FY602 strains, the $h$ mo1 1 nap1 $\Delta$ deletion strain grew better than WT, but the relative growth was no greater than that of individual deletions of HMO1 or NAP1 in the presence of protein VII (Figure 2c and f). Furthermore, we found that protein VII expression levels were largely consistent across strains (Figure 2d and $\mathrm{g}$ ). The observation that simultaneous deletion of HMO1 and NAP1 fails to rescue growth additively supports our hypothesis that these proteins function cooperatively 158 and together give rise to protein VII's impact on growth.

Based on the finding that protein VII expression reduces $\mathrm{H} 1$ chromatin occupancy in human cells (Supplemental Figure 1a) and that protein VII binds linker DNA in vitro ${ }^{32}$, we predicted that 162 protein VII and $\mathrm{H} 1$ compete for the same binding sites on linker DNA. Thus, we expected that 163 deletion of the yeast $\mathrm{H} 1$ homolog, $\mathrm{HHO1}$, would allow for more efficient chromatin disruption by 164 protein VII and a corresponding exacerbation of the protein VII growth defect. In both W303 and 165 FY602, we found that without Hho1 growth was reduced compared to WT (Figure $2 \mathrm{~b}$ and e). 166 While the average relative growth was indeed lower, the reduction was not statistically 167 significant compared to WT (Figure 2c and f). Notably, there were no significant changes in 
168 growth upon GFP expression among the strains tested (Supplemental Figure 4). Therefore, we 169 conclude that the changes in growth caused by gene deletion are specific to protein VII 170 expression. In summary, protein VII growth defects are rescued by the deletion of HMO1, 171 NAP1, or both factors together while the reduction in growth is exacerbated by HHO1 deletion. 172 Taken together, these results support our model in which protein VII interacts with Hmo1 and 173 Nap1 to displace Hho1 and disrupt chromatin resulting in growth defects (Figure 2a).

\section{The human proteins HMGB1 and SET can replace their yeast homologs to facilitate} protein VII's disruption of chromatin in yeast

178 To determine whether the human factors known to bind protein VII could replace the yeast 179 homologs in our system, we introduced the homologous human proteins into corresponding yeast mutant strains and examined their growth. We assayed the outcome of adding human HMGB1 or SET in the corresponding deletion strains by creating plasmids to co-express protein VII and the target human protein from a bidirectional GAL1-10 promoter in both W303 and FY602 strains (Figure 3). As observed above, deletion of HMO1 rescued the protein VII growth defect (Figure 3a-c and g-i). Interestingly, co-expression of HMGB1 with protein VII in the hmo1s strain led to reduced growth, which suggests that HMGB1 can replace Hmo1 in facilitating protein VII's impact on growth. To confirm that these effects on growth were specific to protein VII expression, we tested growth when GFP and HMGB1 were co-expressed in the same set of strains (Supplemental Figure 5a-c and g-i). There was no significant difference in growth when GFP, instead of protein VII, was co-expressed with HMGB1. Thus, we conclude that human HMGB1 can replace yeast $\mathrm{Hmo1}$ to promote the deficient growth caused by protein 191 VII.

We performed the same set of assays using human SET to replace yeast Nap1 and obtained similar results (Figure $3 \mathrm{~d}-\mathrm{f}$ and $\mathrm{j}-\mathrm{I}$ ). NAP1 deletion rescued the protein VII growth defect while expression of human SET in the nap1 $\Delta$ strain restored the protein VII growth deficiency. These observations are consistent with human SET functioning to replace Nap1 in complex with

197 protein VII. When we expressed GFP and SET simultaneously in the nap1 $\Delta$ strain, we found 198 that co-expression did not cause significant changes in growth (Supplemental Figure 5d-f and 199 5j-I). As was the case with HMGB1, replacing Nap1 with SET altered growth only in the 200 presence of protein VII. Consequently, we conclude that human SET can replace yeast Nap1 to 201 promote protein VII growth defects. Together these data produced by expressing protein VII 
with human HMGB1 or human SET support our model in which protein VII interacts with these factors to disrupt chromatin.

\section{Protein VII disrupts the cell cycle in yeast and human cells}

To define the cause of slowed growth in the presence of protein VII, we analyzed cell cycle progression by budding analysis in yeast expressing protein VII. Since the yeast budding cycle is correlated to cell cycle progression and can be halted by cell cycle checkpoint activation, a change in the distribution of budding morphologies during log phase growth can indicate that the cells are unable to progress from one phase of the cell cycle to the next ${ }^{52,53}$. We examined the

212 distribution of bud sizes during mid-log phase growth in liquid culture and found significant over213 representation of cells with large buds when protein VII was expressed compared to the GFP

214 control (Figure 4a). We next analyzed the budding cycle as the cells transitioned from stationary 215 phase to log phase growth during induction of protein VII expression (Figure 4b, Supplemental 216 Figure 6a). As the cells entered log phase growth, the proportion of large-budded cells 217 increased for both protein VII and GFP expressing cells. However, the cells with protein VII 218 accumulated a significantly greater proportion of large-budded cells which persisted over time. 219 Cells with large buds are likely unable to complete the transition from S phase to G2 or from G2 220 to $\mathrm{M}$ at the same rate as normal cells. Thus, we conclude that protein VII expression disrupts 221 the budding cycle which is consistent with cell cycle progression defects. We also performed 222 budding analysis on the same strains without induction of protein expression and found no 223 significant differences (Supplemental Figure 6b and c), indicating that protein VII specifically 224 leads to the accumulation of large-budded cells. Together these results suggest that the 225 chromatin disruption caused by protein VII in yeast leads to cell cycle dysregulation.

227 To assess the impact of protein VII on human cells, we assayed proliferation and cell cycle 228 progression in human diploid retinal epithelial (RPE-1) cells in the presence of protein VII. 229 Because many adenovirus serotypes can cause conjunctivitis ${ }^{54}$, we chose these cells as 230 biologically relevant for adenovirus infection. We expressed GFP-tagged protein VII or a GFP 231 control by a recombinant viral vector then measured cell proliferation and cell cycle progression 232 by flow cytometry to detect DNA content ${ }^{55}$. We found that cells expressing protein VII stopped 233 proliferating immediately, similarly to control cells arrested by treatment with a CDK-1 inhibitor, $234 \mathrm{RO}-3306^{56}$ (Figure 5a; Supplemental Figure $7 \mathrm{a}$ and b). Given that more than $85 \%$ of the protein 235 VII-GFP cells excluded trypan blue stain, we concluded that this loss of proliferation was not 
caused by cell death (Supplemental Figure 7c). Rather, we found that protein VII-GFP expression led to an increase in G2/M DNA-content cells compared to the control (Figure 5b). Since flow cytometry analysis for DNA content does not distinguish between G2 and mitotic cells, we examined phosphorylation of histone $\mathrm{H} 3$ at serine $10(\mathrm{H} 3 \mathrm{~S} 10 \mathrm{ph})$ as a marker of mitotic chromosomes $^{57-59}$. Protein VII-GFP expressing cells had dramatically higher H3S10ph levels compared to the other conditions (Figure 5c). The amount of H3S10ph increased relative to the

242 pre-treatment sample and remained high over time suggesting that the protein VII-GFP cells

243 enter mitosis but are unable to complete it. In agreement with this conclusion, we observed

244 more binucleate cells in the population of protein VII-GFP cells (data not shown). In contrast, the

245 GFP and no treatment samples had a steady decline in H3S10ph which likely reflected the 246 reduction in G2/M cells detected by flow cytometry. The RO-3306-treated cells had nearly

247 undetectable levels of H3S10ph which is consistent with Cdk1-dependent activation of aurora

$248 \mathrm{~A} / \mathrm{B}$ kinases during the G2/M transition ${ }^{60}$. Together, these results indicate that protein VII slows

249 the cell cycle in diploid human cells leading to an enrichment of mitotic cells, which echoes our 250 observations in yeast. Hence, we conclude that expression of protein VII impairs progression of 251 the cell cycle through mitosis.

\section{Protein VII blocks proliferation of HEK 293 cells}

During adenovirus infection, protein VII is expressed from the $\mathrm{L} 2$ late transcriptional unit ${ }^{61,62}$. The finding that ectopically expressed protein VII is a potent inhibitor of proliferation and cell cycle progression in human cells is in stark contrast to the well-studied effects of the early adenovirus genes $\mathrm{E} 1 \mathrm{~A}$ and $\mathrm{E} 1 \mathrm{~B}$, which direct the bypass of cell cycle checkpoints and promote an $S$ phase-like gene expression state ${ }^{31,63}$. This $E 1 A / E 1 B$ gene expression program is the primary driver of transformation in the ubiquitous human cell line HEK $293^{64,65}$. Consequently, we asked whether protein VII could effectively override the pro-proliferation effects of E1A.

To test whether protein VII can abrogate the effects of E1A on proliferation and cell cycle 264 regulation, we generated an E1A/E1B-transformed cell line to express HA-tagged protein VII 265 under a doxycycline-inducible promoter from a single locus using the HILO-RMCE system ${ }^{66}$. 266 After induction of protein VII expression, growth slowed significantly over time compared to the 267 GFP control cells (Figure 5d, Supplemental Figure 7d and e). The loss of proliferation in the 268 inducible protein VII 293 cells occurred more gradually than in the RPE-1 cells likely due to the 269 lower levels of protein VII expressed upon initial induction compared to transduction (Figure 5f). 
270 Like the RPE-1 cells, protein VII expression decreased the number of G1 cells and increased

271 the proportion of G2/M cells (Figure 5e) resulting in an accumulation of H3S10ph (Figure 5f).

272 The growth impairment was not caused by widespread cell death (Supplemental Figure 7f) nor

273 by loss of E1A expression (Figure 5f). Thus, we conclude that protein VII is able to counteract

274 the effects of $\mathrm{E} 1 \mathrm{~A}$ and halt cell proliferation. Together these data suggest that during adenovirus

275 infection, the late-expressed protein VII can counteract the effects of $\mathrm{E} 1 \mathrm{~A}$ on cell cycle 276 regulation and prevent cell division. This newly uncovered role for protein VII suggests that 277 disruption of the cell cycle late in infection may be as critical to viral replication as cell cycle 278 disruption during the earliest phases of infection.

\section{Discussion:}

For nuclear-replicating viruses such as adenovirus, host chromatin is both an impediment to and a bountiful resource for ensuring successful replication. The histone-like protein VII is an important, yet largely mysterious, component of adenovirus' armament for re-purposing host chromatin resources. Previous work showed that protein VII directly interacts with protein $\mathrm{SET}^{67,68}$ and HMGB1 and recruits them to chromatin during infection ${ }^{32}$. Proteomic analysis of chromatin in the presence of protein VII revealed that linker histones are depleted while SET and HMGBs are enriched, suggesting that protein VII may co-opt these host factors to disrupt linker histone occupancy ${ }^{37,69,70}$. As such, we hypothesized that protein VII exploits the antagonism between $\mathrm{H} 1$ and HMGB1 to undermine chromatin structure and maximize viral success. Here, we coupled budding yeast genetics and human cell experiments to test our model of linker histone displacement and chromatin disruption via SET and HMGB proteins.

The expression of protein VII elicited a significant growth defect in two separate lab yeast strains, demonstrating the robustness of protein VIl's chromatin perturbation. Protein VII expression led to smaller colony size, indicating slowed growth, while fewer colonies grew on the solid media suggesting a loss of viability or proliferation (Figure 1). This is consistent with

298 the large-budded cell phenotype observed during exponential growth (Figure 4) and suggests 299 that cell cycle checkpoint activation leads to cell death or reduced growth rate. As a control, we 300 expressed the VII- $\triangle$ PTM protein which, in human cells, cannot be post-translationally modified 301 and therefore no longer localizes to chromatin. In yeast, expressing VII- $\triangle \mathrm{PTM}$ did not cause 302 growth defects suggesting that these sites are also critical for protein VIl's disruption of 303 chromatin in yeast. It is possible that protein VII is modified in yeast and that these modifications 
regulate protein VII's interaction with and impact on chromatin. It may also be the case that structural changes caused by the mutations render protein VII unable to impact chromatin despite stable expression. While protein VII bound chromatin across the yeast genome and thus was not significantly enriched at specific loci, the genomic profile is distinct from that of total $\mathrm{H} 3$ (Supplemental Figures 2 and 3). Further work focused on protein VII occupancy will define the specificity of protein VII binding genome wide.

Deleting HMO1 or NAP1, the yeast homologs for HMGB1 and the histone chaperone protein SET, rescued the growth defects caused by protein VII expression while deletion of the linker histone, HHO1, exacerbated this phenotype. Interestingly, HMO1 deletion had a more profound effect in W303 while NAP1 deletion led to greater rescue in FY602 (Figure 2). Furthermore, when both factors were deleted, the magnitude of rescued growth matched that of a single gene deletion. For example, in W303, the relative growth of the hmo1 1 nap $1 \Delta$ strain was roughly equal to that of the $h m o 1 \Delta$ strain. The lack of an additive rescue indicates that these factors function in the same genetic pathway with respect to protein VII. However, the strain-specific results suggest that the relationship between $\mathrm{Hmo1}$ and protein VII has a greater impact in W303, while protein VII's effect is mediated more by Nap1 in FY602. These unique strain relationships are also evident upon re-introduction of the human homologs. For both strains, expressing the homologous human factors when protein VII was present rescued the growth phenotype in the corresponding deletion strains (Figure 3). However, expression of HMGB1 in 324 the W303 hmo1 $\Delta$ strain had a greater impact on growth than the expression of protein SET in 325 the nap1 $\Delta$ strain. We observed the opposite relationship in FY602. Given that W303 has nearly 9500 single nucleotide variations affecting nearly $12 \%$ of all genes when compared to $\mathrm{FY} 602^{71}$, 327 it is likely that this considerable variation between the two strains gives rise to the different 328 apparent preference for Hmo1 or Nap1. In neither case, however, did expression of the human 329 factor completely rescue the protein VII growth defect suggesting that other factors contribute to 330 protein VII's disruption of chromatin.

332 Consistent with our model that protein VII and $\mathrm{H} 1$ compete for the same binding sites, deletion 333 of the linker histone exacerbated the effects of protein VII on yeast growth (Figure 2). As such, $334 \mathrm{H} 1$ may protect chromatin from protein VII invasion. Based on the protein VII ChIP-seq from 335 yeast chromatin (Supplemental Figures 2 and 3), we propose that protein VII binds human 336 chromatin ubiquitously, without preference for DNA sequence or chromatin state. However, 337 locations that have higher $\mathrm{H} 1$ occupancy may be resistant to protein VII binding, and thus 
protein VII requires the action of protein SET and HMGB proteins to facilitate its chromatin invasion. This would explain why the effects of HHO1 deletion in yeast are relatively slight with respect to protein VII growth impairment. Compared to human cells, yeast have very little heterochromatin which is silenced by means other than linker $\mathrm{H} 1$ binding $^{72}$. Therefore, a large portion of the yeast genome is vulnerable to protein VII invasion, even without HHO1 deletion, compared to the human genome which is thought to be comprised of $\sim 25 \%$ heterochromatin ${ }^{73}$.

344 Furthermore, the post-translational modifications of $\mathrm{H} 1$ proteins, which have not been well 345 characterized, may regulate $\mathrm{H} 1$ localization and antagonism of HMG proteins. Given that post-

346 translational modification of protein VII impacts its relationship with chromatin, it is possible that protein VII competes for these $\mathrm{H} 1$-modifying enzymes to cause chromatin dysregulation in yet another dimension.

Similar to our observations in yeast, protein VII resulted in a dramatic loss of proliferation and cell cycle arrest in diploid RPE-1 cells (Figure 5). The block of cell growth and shift in cell cycle profiles occurred rapidly, within 12 hours of protein VII expression. However, the block of growth took longer in the HEK 293 cells likely due to lower levels of protein VII expression driven from a

354 single copy of the gene as opposed to the multiple copies introduced to each RPE- 1 cell by transduction. While the most profound effects on cell cycle progression seemed to occur during mitosis, we also observed an increased $S$ phase population in both human cell types. This buildup of S phase cells suggests that protein VII's interruption of cell cycle progression can occur at multiple points.

In HEK 293 cells, E1A promotes proliferation and transforms the cells by re-wiring their basal epigenetic state. This occurs through redistributing $\mathrm{H} 3$ acetylation which then alters global gene expression $^{26,74}$. E1A most significantly upregulates acetylation of $\mathrm{H} 3 \mathrm{~K} 18$ to reprogram transcription ${ }^{1}$. In contrast, protein VII expression does not cause significant changes to $\mathrm{H} 3 \mathrm{~K} 18 \mathrm{ac}$ or most other histone marks ${ }^{32}$. This suggests that the mechanism of protein VIl's block of proliferation in HEK 293 cells is not through changes to histone modifications. Likewise, although protein VII and E1A have been shown to directly interact ${ }^{75}$, the effects of protein VII on

367 proliferation and cell cycle regulation occur through means independent of this interaction given 368 that these effects are observed in cells without E1A expression (Figure 5) and in budding yeast 369 (Figure 1). 
371 It is remarkable that, although budding yeast and humans are separated by one billion years of

372 divergent evolution and yeast have not encountered adenovirus infection during that

373 evolutionary history, adenovirus protein VII disrupts yeast chromatin just as it does human

374 chromatin. This finding underscores both the conservation of chromatin biology across

375 eukaryotes as well as the robust and catastrophic effects of protein VII on the most basic

376 functions of chromatin. Our study, as well as others ${ }^{76}$, demonstrates the power of yeast to study

377 the effects of histone-like proteins on chromatin. In light of this new role for protein VII in

378 undermining cell cycle progression, we propose that protein VII expression late in the infection

379 cycle maximizes progeny production by counterbalancing checkpoint bypass and transcriptional

380 reprogramming initiated by the early-expressed factors E1A and E1B (Figure 5g). These earliest

381 expressed adenovirus proteins induce a "viral S phase"77,78 while a complement of other early

382 adenovirus proteins inactivate the host DNA damage response ${ }^{79}$. Since both the intra-S phase

383 and G2 host cell cycle checkpoints rely on sensing DNA damage to stall cell cycle progression,

384 it is conceivable that, as a consequence of this un-checked "viral S phase", an infected cell

385 could initiate mitosis and cytokinesis before viral progeny assembly and release. In this

386 scenario, breakdown of the nucleus would disrupt progeny assembly and cell division would

387 redirect nuclear resources away from viral replication resulting in reduced progeny. Given the

388 effects of protein VII on mitosis, we propose that protein VII prevents an infected cell from

389 initiating cytokinesis, therefore maximizing virion production. Studies of several mutant

390 adenoviruses provide indirect evidence that late viral proteins can prevent the onset of mitosis.

391 For example, cells synchronized in early G1 then infected with mutant adenovirus lacking E1B-

$39255 \mathrm{k}$ and E4orf3 are predisposed to arrest in a mitosis-like state late in infection ${ }^{78}$. It's likely that

393 mutant viruses do not highly express late proteins, including protein VII. Therefore, given our

394 observation that protein VII impedes mitosis without the need for other early adenovirus

395 proteins, it will be informative to test the contribution of protein VII and its binding partners to cell

396 cycle progression during infection.

398 In sum, we show that adenovirus protein VII disrupts chromatin by exploiting H1-HMGB 399 antagonism and, as a result, impairs cell cycle progression. Our findings in yeast and human 400 cells reveal a highly conserved chromatin vulnerability that adenovirus exploits, underpinning 401 the evolutionary conservation of H1-HMGB antagonism. HMGB1 has long been the focus of 402 studies of host inflammatory responses ${ }^{80,81}$, but its intranuclear role during infection is also 403 intimately linked to viral infection ${ }^{32}$. Intriguingly, HMGB1 was recently found to be required for 404 SARS-CoV-2 infection ${ }^{82}$. Although the mechanism is not known, preliminary evidence suggests 
405

406

407

408

409

410

411

412

413

414

415

416

417

418

419

420

421

422

423

424

425

426

427

428

429

430

431

432

433

434

435

436

437

that the interaction of HMGB1 with chromatin underlies this dependency, which hints that the novel vulnerability we expose in H1-HMGB1 antagonism may be exploited by a wide range of intracellular pathogens.

\section{Acknowledgements}

We thank members of the Avgousti lab, M. Emerman, M. Weitzman, E. Hatch, S. Parkhurst and T. Tsukiyama for insightful comments on the manuscript. We also thank $\mathrm{H}$. Wodrich for generously providing anti-VII antibodies and D. Curiel for sharing recombinant adenoviruses. We also thank N. Donadio for technical help, the Hatch lab for assistance with cell cycle dynamics, the Brewer/Raghuraman lab for providing yeast strains, L. Howe for providing yeast strains and constructs, and the Tsukiyama lab for invaluable advice, reagents and insight for yeast and chromatin experiments. We also thank the Fred Hutch Cellular Imaging Shared Resource for assistance with microscopy and image analysis. This research was supported by the Cellular Imaging Shared Resource (CISR) and Genomics Core Facility of the Fred Hutch/University of Washington Cancer Consortium (P30 CA015704). We also thank the Bioinformatics and Flow Cytometry Facilities at Fred Hutchinson Cancer Research Center for their assistance. The research presented here was supported in part by start-up funds from Fred Hutchinson Cancer Research Center and NIH funding to DCA (R35-GM133441), KLL (T32-CA009657), and HCL (T32-AI083203).

\section{Author Contributions}

Conceptualization, K.L.L, D.C.A.; Methodology, K.L.L, D.C.A.; Formal Analysis, K.L.L., M.B., M.R.D.; Investigation, K.L.L., M.B., M.R.D., H.C.L., D.C.A.; Resources, K.L.L., M.B., M.R.D., H.C.L., D.C.A.; Data Curation, K.L.L., M.B., M.R.D.; Writing - Original draft, K.L.L and D.C.A.; Writing - Review \& Editing, K.L.L., M.B., M.R.D., H.C.L., D.C.A.; Visualization, K.L.L., M.B., M.R.D.; Supervision, K.L.L, D.C.A.; Funding Acquisition, K.L.L, D.C.A.

\section{Conflict of Interests}

The authors declare that no conflicts of interest exist.

\section{Materials and Methods}

\section{Yeast Expression Plasmids}


438 We used a standard GAL1 promoter plasmid and inserted a protein VII sequence codon 439 optimized using the IDT algorithm for expression in S. cerevisiae. To do so, we synthesized 440 gBlock Gene Fragments (Integrated DNA Technologies) corresponding to mature protein VII 441 from human adenovirus type 5 and a version of the same protein coded for alanine substitutions 442 at five residues previously found to be post-translationally modified ${ }^{32}$. Both versions of protein 443 VII were encoded with three consecutive HA epitope tags at the C terminus. These protein VII 444 DNA fragments, as well as yeast codon-optimized GFP sequence from the plasmid PFA6A445 GFP-KanMx ${ }^{83}$, were PCR amplified to have 3' and 5' Spel and HindIII restriction sites 446 respectively. The amplified sequences were then cloned and confirmed by Sanger sequencing 447 across both junctions. To generate the co-expression plasmids with either WT protein VII or 448 GFP, the GAL1 promoter was first replaced with the full-length bidirectional GAL1-10 that was 449 PCR amplified from the FY602 strain and engineered with Xbal and Sacl restriction sites. We 450 confirmed successful replacement with the bidirectional GAL1-10 promoter by Sanger 451 sequencing. Next, we PCR amplified human protein SET or human HMGB1 DNA fragments 452 (codon optimized for yeast) containing Sacl restriction sites at both ends of the fragments from 453 plasmids synthesized by GeneWiz. These fragments were then cloned opposite either GFP or 454 protein VII allowing for co-expression of either gene with human protein SET or HMGB1.

\section{Yeast Strain Construction and Maintenance}

457 We obtained WT FY602 from from Dr. Leann Howe at the University of British Columbia ${ }^{76}$ and 458 introduced gene deletions for HMO1, HHO1, and NAP1 using one-step homologous 459 recombination gene replacement as in reference ${ }^{84}$. The HIS3 gene used for replacement of $460 H M O 1$ was amplified from pRS413 ${ }^{85}$ and the KanMx6 gene used for replacement of HHO1 or 461 NAP1 was amplified from pUG6 ${ }^{86}$. WT W303 was obtained from the Brewer-Raghuraman lab 462 and all W303-derived deletion strains were obtained from the Tsukiyama lab. The linear deletion 463 fragments or plasmids were introduced by standard lithium acetate transformation ${ }^{87}$ and growth 464 on selective media. Transformed colonies were streaked out for single isolates on selective 465 media and candidate gene deletions were confirmed by diagnostic PCR to confirm integration of 466 the selection gene as well as Sanger sequencing of the insertion junctions. For strains obtained 467 from other labs, putative gene deletions were confirmed by diagnostic PCR to confirm 468 integration of the selection gene. Expression plasmids were transformed into relevant strains 469 every four weeks. Colonies were streaked onto selective media, grown at $30^{\circ} \mathrm{C}$ until single 470 colonies formed, and then stored at $4^{\circ} \mathrm{C}$. Strains containing the expression plasmids were 471 maintained in selective media for all pre-growth steps. 


\section{Yeast Media}

474 Yeast media was prepared using ultrapure water (Millipore Sigma Synergy UV Remote Water

475 Purification System, \#SYNSVROWW) and sterilized with 15-minute liquid autoclave cycle.

476 Standard rich media (YEP) was prepared with bactopeptone (Fisher \# DF0118-17-0), yeast

477 extract (Fisher \#BP1422-500), and 2\% dextrose (Fisher Bioreagents \#D16-500) with 2.4\% agar

478 (Fisher Bioreagents \#BP1423-500) for agar plates. Kanamycin was added to $400 \mathrm{mg} / \mathrm{mL}$ in

479 YEPD plates for selection. Complete media without uracil (SC-ura) or histidine (SC-his) was

480 purchased from Sunrise Science Products (\#1306-030 and \#1303-030) and prepared per the

481 manufacturer's instructions with yeast nitrogen base (\#1501-250) and with 2.4\% agar for solid

482 media plates. To derepress the galactose-promoter, cells were grown in SC-ura $+2 \%$ raffinose

483 media (Fisher \#AC195675000). The induce protein expression, cells were grown in SC-ura +

$4842 \%$ galactose media (Fisher \#BP656-500).

\section{Yeast Growth Assays and Quantification}

487 For serial dilution growth assays, plasmid-bearing strains were pre-grown for 24 hours in SC-ura

$488+2 \%$ dextrose liquid media at $30^{\circ} \mathrm{C}$ with agitation. Cultures were sonicated at $35 \%$ amplitude for

4897 seconds using a Fisher Scientific FB50 Sonic Dismembrator to disaggregate the cells before

490 dilution. Cells were diluted in ultrapure water and counted by hemocytometer, then six 1:3 serial

491 dilutions were prepared in a 96-well plate such that the most dilute cell mixture contained 3

492 cells/uL. After vigorous trituration, $5 \mathrm{uL}$ of each solution was placed onto SC-ura $+2 \%$ dextrose

493 agar media to repress protein expression or SC-ura $+2 \%$ galactose agar media to induce 494 protein expression. The plates were dried at room temperature and then incubated at $30^{\circ} \mathrm{C}$ for 495 six days. Each day, the plates were imaged on a BioRad ChemiDoc MP Imaging System using 496 the "Ponceau S" setting and 0.5s exposure time. The images were processed using Adobe 497 Creative Cloud Photoshop and Illustrator. The serial dilution assays were quantified using 498 BioRad Image Lab v6.1 software. Using the Volume Tools analysis package, a box with the 499 same pixel dimensions was drawn around the third position in the dilution series (see Figure 1) 500 for both the dextrose- and galactose-grown cells and the signal intensity values were measured 501 for each position independently. The local subtraction method was used to remove background 502 signal. To determine the relative growth value, the signal intensity of grown on galactose media 503 was divided by the signal intensity from dextrose media growth. For WT, nap1 $\Delta$ and hho1 $\Delta$ 504 strains with the single protein expression plasmids, which all had the same growth rate, the 505 relative growth values were calculated from the images taken after three days growth at $30^{\circ} \mathrm{C}$. 
506 To compensate for slowed growth caused by Hmo1 deletion, the relative growth for hmo1s and

507 hmo14nap1A strains was measured after four days growth. For strains with the co-expression 508 plasmids, which had slightly different growth rates, relative growth was calculated after five days 509 growth during GFP expression or six days growth during protein VII expression. Quantification 510 of the serial dilution assays represent at least three independent replicate experiments. 511 Statistical tests were carried out as described in the figure legends using GraphPad Prism v9.0 512 software.

514 For the exponential growth analysis, strains were pre-grown for 24 hours in SC-ura $+2 \%$ 515 dextrose liquid media at $30^{\circ} \mathrm{C}$ with agitation, then scaled up to a larger culture volume in SC-ura $516+2 \%$ raffinose liquid media and grown for another 24 hours. To start the exponential growth 517 experiments, the raffinose-grown cultures were diluted to $\mathrm{OD}_{660}$ of 0.15 in SC-ura $+2 \%$ dextrose 518 or SC-ura $+2 \%$ galactose and incubated at $30^{\circ} \mathrm{C}$ with agitation. The $\mathrm{OD}_{660}$ was measured every 519 hour and graphs were generated in real time. Western blot samples for protein expression 520 confirmation were collected at 4-, 6-, 8- and 10-hours post-induction for the first experimental 521 replicate and at 6 hours post-induction for follow-up experiments. To determine exponential 522 growth rate, the $\mathrm{OD}_{660}$ was graphed on a log-10 scale and an exponential growth equation with 523 least squares fit was fit to at least five datapoints using GraphPad Prism v9.0 software. The bar 524 charts represent the average exponential growth value $(k)$ from at least three biological replicate 525 experiments. Statistical tests are described in the figure legends and were performed with 526 GraphPad Prism v9.0 software.

\section{Yeast Western Blotting}

529 To confirm protein expression, western blot samples were recovered after six hours induction or 530 repression during exponential growth. To equalize the number of cells in each sample, culture 531 volume containing $2 \mathrm{OD}_{660}$ of cells was centrifuged to pellet the cells. The cells were washed 532 once with filtered purified water, pelleted again, and the dry pellets were stored at $-80^{\circ} \mathrm{C}$ until 533 processing. To prepare whole cell lysate, the cell pellet was resuspended in $100 \mathrm{uL}$ water then $534100 \mathrm{uL} 0.2 \mathrm{M} \mathrm{NaOH}$ was added and cells were incubated at room temperature for five minutes ${ }^{88}$. 535 The cells were pelleted, then resuspended in SUMEB loading buffer (1\% SDS, 8 M Urea, 10 $536 \mathrm{mM}$ MOPS, pH 6.8, 10 mM EDTA, 0.02\% bromophenol blue) with protease inhibitor cocktail 537 (MilliPore Sigma \#11836170001) and 5\% 2-mercaptoethanol followed by heating at 65ㄷ for 10 538 minutes. Samples were loaded onto 15\% SDS-PAGE gels and run via standard methods in 539 NuPAGE MOPS SDS running buffer (Invitrogen \#NP0001). Gels were transferred (Transfer 
540 Buffer: $25 \mathrm{mM}$ Tris Base, $100 \mathrm{mM}$ glycine, 20\% methanol) onto nitrocellulose membrane and

541 Ponceau stained, blocked with 5\% milk for 30 minutes, and stained with primary antibody

542 overnight. Primary antibodies and dilutions used were: $\alpha-G F P$ (1:2500; Abcam \#290), a-HA

543 (1:500; BioLegend \#MMS-101R), a-PGK1 (1:5000; Abcam \#113687), and a-H3 (1:2500; Abcam

544 \#1791). After standard washing, blots were probed with either a-mouse (1:5000; Jackson

545 ImmunoResearch \#115-035-003) or a-rabbit (1:5000; Jackson ImmunoResearch \#111-035-045)

546 HRP-conjugated secondary antibody and developed with ECL per the manufacturer's

547 instructions (BioRad Clarity ECL \#1705061). Images were acquired using the BioRad

548 ChemiDoc MP Imaging System.

550 Yeast Immunofluorescence

551 Cultures were pre-grown in liquid SC-ura $+2 \%$ raffinose media as previously described, then 552 scaled to $50 \mathrm{~mL}$ culture volume at an $\mathrm{OD}_{660}$ of 0.3 in SC- SC-ura $+2 \%$ dextrose or SC-ura $+2 \%$ 553 galactose liquid media. Cultures were incubated for 4 hours at $30^{\circ} \mathrm{C}$ with agitation. After 554 induction, culture $\mathrm{OD}_{660}$ was measured such that equivalent numbers of cells could be collected 555 by centrifugation. Cells were sonicated for 10 seconds at $50 \%$ duty cycle, output 2 using a 556 Branson Sonifier 250 Model SSE-1 probe sonicator (\#510-294-301). The cells were pelleted by 557 centrifugation at 3000 rpm for 5 minutes, washed twice in water, resuspended in $1 \mathrm{~mL} 4 \%$ PFA 558 diluted PBS, and incubated for 1 hour at room temperature on a nutating platform mixer to fix 559 the cells. Cells were pelleted by centrifugation as before, washed and then resuspended in $1 \mathrm{~mL}$ 560 Wash Buffer (0.1 $\mathrm{M} \mathrm{KH}_{2} \mathrm{PO}_{4}$ in $1.2 \mathrm{M}$ sorbitol solution). Cells were counted by hemocytometer 561 and 40 million cells were moved to a fresh microtube and brought up to $200 \mathrm{uL}$ total volume in 562 Wash Buffer. Zymolyase (10 mg/mL Zymo Research \#ZE1005) and 2-mercaptoethanol was 563 added to the cells which were incubated for 50 minutes at $30^{\circ} \mathrm{C}$ to spheroplast the cells. Cells 564 were washed twice by pelleting then resuspension in $1 \mathrm{~mL}$ Wash Buffer. Cells were then 565 resuspended in $200 \mathrm{uL} 3 \%$ BSA solution in PBS and incubated at room temperature with 566 agitation for $30 \mathrm{~min}$. Cells then incubated in primary antibody solution containing a-HA (1:400; 567 Abcam \#9110) and a-PGK1 (1:400; Abcam \#113687) antibodies in the 3\% BSA solution, then 568 incubated for 1 hour with rocking at room temperature out of direct light. Cells were washed 569 thrice in 3\% BSA solution, then suspended in $200 \mathrm{uL}$ secondary antibody solution containing a570 rabbit Alexa Fluor 488 (1:300; Invitrogen \#A11008), a-mouse Alexa Fluor 555 (1:300, Invitrogen 571 \#A28180), and $2 \mathrm{ug} / \mathrm{mL}$ DAPI (Fisher \#50-874-10001). Cells were washed once in 3\% BSA 572 solution, three times in PBS, and resuspended in $50 \mathrm{uL}$ PBS. Cells were counted by 573 hemocytometer and diluted to a final concentration of 500,000 cells per uL in PBS. 2 uL of cell 
574 solution was mixed with Invitrogen ProLong Gold Antifade (\#P36934) and mounted on poly-

575 lysine slides. Slides were visualized with a Zeiss 780 LSM confocal microscope, maximum

576 intensity projection images were processed in FIJI and assembled using Adobe Photoshop and

577 Illustrator.

\section{Yeast Chromatin Purification and Native Chromatin Immunoprecipitation (ChIP-seq)}

580 After 6 hours galactose induction during log-phase growth in liquid culture, $500 \mathrm{~mL}$ culture 581 volume of cells was collected, nuclei purified, chromatin isolated, and native ChIP performed as 582 previously described ${ }^{89}$. The ChIP protocol was performed using $\alpha-\mathrm{H} 3$ (Abcam \#1791) and $\alpha-$ 583 protein $\mathrm{VII}^{44}$ antibodies and Invitrogen Protein G Dynabeads for Immunoprecipitation 584 (ThermoFisher catalog \#10004D). Libraries were prepared from the ChIP samples as well as a $58510 \%$ input, following manufacturer recommendations, with KAPA Biosystems Unique Dual586 Indexed Adapter Kit (\#KK8727), Hyper Prep Kit (\#KK8504), and Beckman Coulter AMPure XP 587 Beads (\#A63880) for library cleanup and size selection. Library concentrations were measured 588 with Invitrogen Qubit dsDNA HS Assay Kit (\#Q32851), then analyzed with Agilent High 589 Sensitivity D5000 ScreenTape System, and pooled. Libraries were sequenced with 50-bp 590 paired-end reads on an Illumina NovaSeq 6000 SP sequencer at the Fred Hutch Genomics 591 Core Facility. Reads were aligned to the Saccer3 yeast genome assembly (GenBank accession 592 GCA_000146045.2) using Bowtie2. Read coverage was assessed using bamCoverage 593 package from DeepTools2.0. Reads aligning uniquely to the yeast genome were used to identify 594 protein VII peaks using the MACS2 peak caller. Peaks designated as "narrow" and bedGraph 595 tracks showing read coverage for the input, H3, and protein VII ChIP samples for one replicate 596 are shown in Supplemental Figures 2 and 3. ChIP-seq was performed in duplicate. All ChIP-seq 597 sequencing data are available at GEO accession \#GSE164684.

\section{Yeast Budding Index}

600 FY602 strains were pre-grown in raffinose and transferred to SC-ura $+2 \%$ dextrose or SC-ura + $6012 \%$ galactose liquid media as during exponential growth analysis described above. Samples 602 were collected every 1-2 hours during growth/induction. $500 \mathrm{uL}$ of each culture was pelleted, 603 washed once in MilliQ water, resuspended in $70 \%$ ethanol, and fixed overnight at $4^{\circ} \mathrm{C}$. Samples 604 were pelleted, washed once with $50 \mathrm{mM}$ sodium citrate, resuspended and diluted in the same 605 solution. Cells were sonicated as described above and then loaded onto a hemocytometer 606 counting chamber. 120-170 cells per sample were classified as "unbudded", "small-budded" 607 (bud < 50\% the size of the mother cell), "large-budded" (bud > 50\% the size of the mother cell), 
608 or "irregular" (multiple buds or other irregularities). Data were graphed and statistical analyses

609 performed as described in the figure using GraphPad Prism v9.0 software.

\section{Human Cell Line Generation and Maintenance}

612 hTERT-immortalized RPE-1 cells were obtained from Dr. Emily Hatch at Fred Hutchinson 613 Cancer Research Center and were grown in 1:1 DMEM/F12 media (Thermo Fisher \#11330032) 614 with 10\% FBS (Sigma \#FO926-500ML; Lot \#18C539), 1\% penicillin-streptomycin (Fisher \#15615 140-122), and 0.01\% hygromycin (Sigma \#H3274-50MG). HEK293T HILO acceptor cells were

616 obtained from Dr. Eugene Makeyev. Cell lines containing either the GFP reporter or HA-tagged 617 protein VII under a doxycycline-inducible promoter were generated as previously reported ${ }^{32,66}$. 618 After plasmid transfection and puromycin selection, HEK293T HILO cell lines were grown in 619 DMEM (Fisher \#11965118) with tetracycline-free 10\% FBS (Gemini Bio-Products \#100-800; Lot 620 \#A69G00J), 1\% penicillin-streptomycin (Fisher \#15-140-122) and $1 \mathrm{ug} / \mathrm{mL}$ puromycin (Fisher 621 \#MIR5940) to maintain selection.

\section{Exogenous Protein Expression in Human Cells}

624 RPE-1 cells were plated at $20 \%$ confluence in 6-well plates and the following day, cells were 625 transduced with recombinant adenovirus with the $\mathrm{E} 1$ region replaced with either GFP-tagged 626 protein $\mathrm{VII}(\mathrm{MOI}=50)$ or a GFP control $(\mathrm{MOI}=50)$ under a CMV promoter as previously 627 described $^{90}$. Control cells were treated with $10 \mu \mathrm{M}$ RO-3306 (Sigma-Aldrich \#SML0569-5MG). 628 For all treatment groups, samples were collected every 12 hours post-treatment for 48 hours 629 total as described below. The HEK293T doxycycline-inducible cell lines were removed from 630 puromycin selection and plated at a density of $2.6 \times 10^{4}$ cells $/ \mathrm{cm}^{2}$ in $60 \mathrm{~mm}$ plates with 0.2 $631 \mu \mathrm{g} / \mathrm{mL}$ doxycycline to induce protein expression. All cell lines and treatment groups were 632 imaged, and samples were collected every 24 hours post-treatment for 96 hours. Cells were 633 imaged using Leica Microsystems DM IL LED Fluorescent microscope with Leica Application 634 Suite V4.12 software. Images were processed using FIJI, Adobe Photoshop, and compiled with 635 Adobe lllustrator.

\section{Human Cell Proliferation Assay}

638 RPE-1 cells were harvested every 12 hours after transduction for 48 hours in addition to a pre639 transduction sample. HEK293T-based cells were harvested every 24 hours after plating and 640 induction up to 96 hours. For each collection, both cell types were lifted by trypsinization. Cells 641 were pelleted by centrifugation (1200 rpm for 2 minutes) and then suspended in $1 \mathrm{~mL}$ DPBS +1 
$642 \mathrm{mM}$ EDTA to prevent clumping. $20 \mathrm{uL}$ cell suspension was diluted $1: 1$ with $0.4 \%$ Trypan Blue

643 solution (Bio-Rad \#1450021) and cells were counted by hemocytometer (RPE-1 cells) or using

644 a BioRad TC20 Automated Cell Counter (HEK293T-based cells lines). The total number of cells

645 in each well and the number of trypan negative cells was reported.

\section{Human Cell Flow Cytometry}

648 Cells were lifted with trypsin, pelleted by centrifugation (1000 rpm for 5 minutes at $4^{\circ} \mathrm{C}$ ), and 649 washed in $5 \mathrm{~mL}$ FACS Buffer (DPBS + 0.1\% BSA). Cells were pelleted and resuspended in 1 $650 \mathrm{~mL}$ 0.5\% PFA diluted in DPBS and incubated for 5 minutes at room temperature to fix the cells. 651 Cells were washed twice in FACS Buffer and kept on ice or at $4^{\circ} \mathrm{C}$ for all remaining processing. 652 Cells were then suspended in $70 \%$ ethanol and stored at $-20^{\circ} \mathrm{C}$ overnight. Next, the cells were 653 washed three times in DPBS $+0.1 \%$ Triton-X, then resuspended in $500 \mathrm{uL}$ DPBS $+0.2 \mathrm{mg} / \mathrm{mL}$ 654 RNAse A (Sigma \# 10109142001) and $20 \mathrm{ug} / \mathrm{mL}$ propidium iodide (Fisher \# AC440300250), 655 then incubated in the dark at $4^{\circ} \mathrm{C}$ for at least 3 hours. Cells were run on a BD Biosciences 656 FACSCanto II Cell Analyzer in the Fred Hutchinson Cancer Research Center Flow Cytometry 657 core facility. Since GFP was present in $>90 \%$ of the cells expected to express GFP, cells were 658 not gated based on GFP. Instead, single cells were gated using propidium iodide area and 659 height measurements. Histograms indicating DNA content were generated using FlowJo v10 660 software.

661

\section{Human Cell Western Blotting}

663 Western blot cell samples were counted and then pelleted and resuspended in 1x NuPAGE 664 LDS sample buffer (Fisher \#NP0007) + 5\% 2-betamercaptoethanol. The mixture was heated for 66520 minutes at $95^{\circ} \mathrm{C}$ and stored at $-20^{\circ} \mathrm{C}$. $10 \mathrm{uL}$ of each sample was run on a $15 \%$ SDS PAGE 666 gel using standard methods as described above with the following primary antibodies: $\alpha$-vinculin 667 (1:20,000 Sigma \#V9131), a-GFP (1:2500, Abcam \#290), a-H3 (1:20,000, Abcam \#1791, a668 H3S10ph (1:1000, EMD Millipore \#06-570), a-HA (1:500, BioLegend/Covance \#MMS-101R) or 669 a-E1A (1:250, BD Biosciences \#554155); and secondary antibodies: a-mouse (Jackson 670 ImmunoResearch \#115-035-003) or a-rabbit (Jackson ImmunoResearch \#111-035-045). Blots 671 were developed with Clarity Western ECL (Bio-Rad \#1705061) and images were processed as 672 described above.

673 
a.

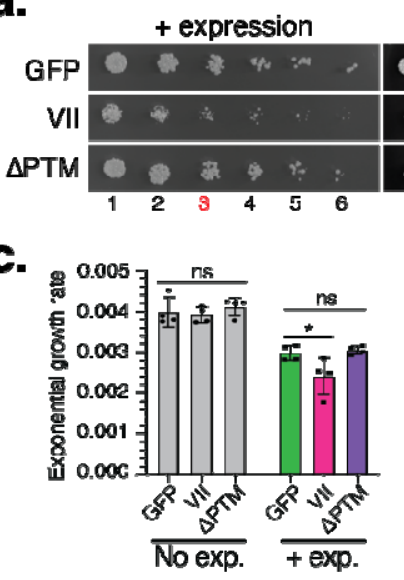

e. No expression b.

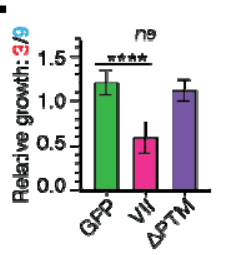

d.

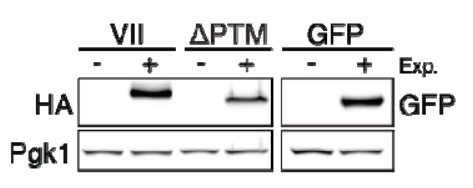


691 significance was found among the strains without protein expression (GFP vs. VII $p=0.9420$;

692 GFP vs. VII- $\triangle \mathrm{PTM} p=0.7830$ ). Cells expressing VII had a significantly reduced growth rate 693 compared to GFP ( $p=0.0157)$ while cells expressing VII- $\Delta$ PTM did not $(p=0.9616)$. Error bars

694 show SD. $\mathrm{N}=4$. d. Western blot analysis of WT W303 strains with and without protein 695 expression during exponential growth. Protein VII and VII- $\triangle$ PTM are identified with an HA tag 696 and GFP indicates the control strain. Phosphoglycerate kinase (Pgk1) is the loading control. e. 697 Representative serial dilution growth assay for growth of WT FY602 with and without GFP, 698 protein VII, or VII VII- $\triangle \mathrm{PTM}$ expression as described in (d). f. Quantification of WT FY602 serial 699 dilution growth assays as described in (e). Cells expressing protein VII grew $\sim 65 \%$ less than 700 GFP, which was statistically significant ( $p<0.0001$, one-way ANOVA with multiple comparisons), 701 while cells expressing VII- $\triangle$ PTM mutant had $\sim 10 \%$ less growth than GFP $(p<0.0001$, one-way 702 ANOVA with multiple comparisons). Error bars denote $\mathrm{SD} . \mathrm{N}=18$. g. Exponential growth rates 703 measured from WT FY602 with or without protein expression. Using 2-way ANOVA for multiple 704 comparisons, no statistical significance was found among the uninduced strains compared to 705 GFP control (GFP vs. VII $p=0.9651$; GFP vs. VII- $\triangle$ PTM $p=0.3129$ ). Cells expressing protein VII 706 had significantly reduced growth rate compared to GFP $(p=0.0098)$ while cells expressing VII$707 \triangle \mathrm{PTM}$ did not $(p=0.9865)$. Error bars show SD. $\mathrm{N}=3$. h. Western blot analysis of WT FY602 with 708 and without protein expression during log phase growth. Protein VII and VII- $\mathrm{PT}$ TM are identified 709 with an HA tag, GFP indicates the control strain, and Pgk1 is the loading control. 
a.

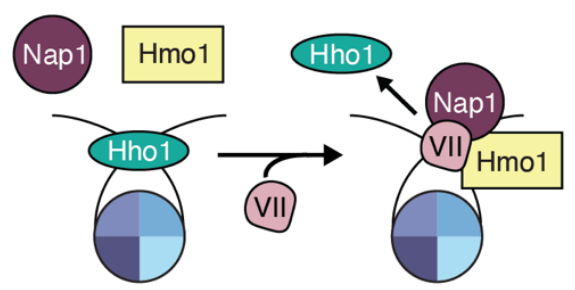

b.

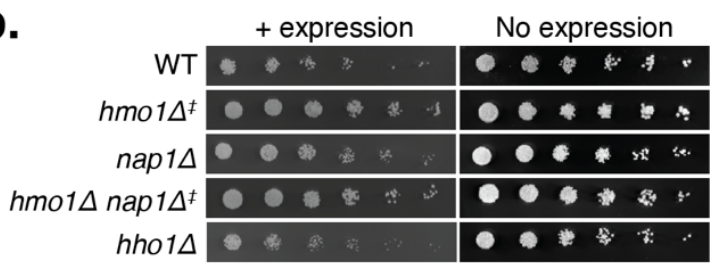

C.

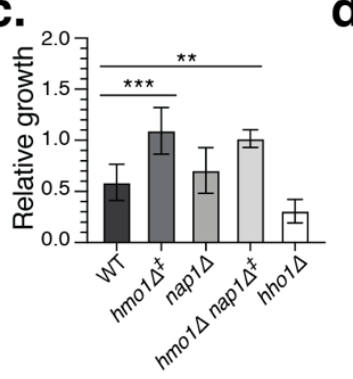

d.

e.

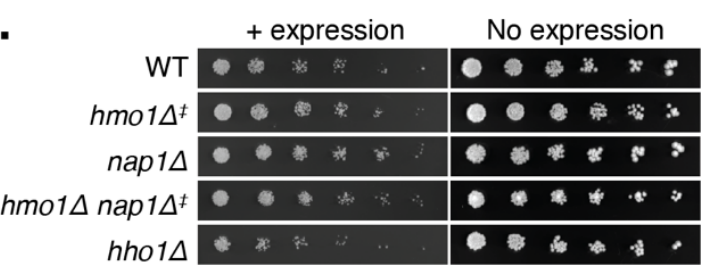

f.

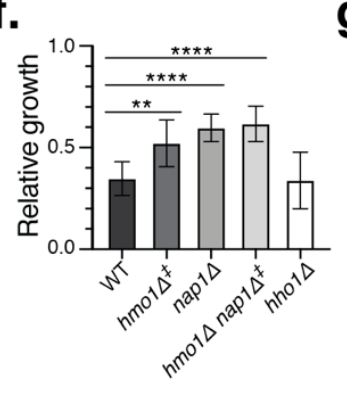

g.

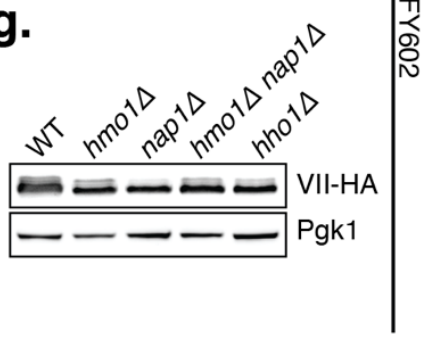

714 Figure 2. The loss of binding partners Hmo1 or Nap1 rescues protein VII growth defects.

715 a. Model for protein VII chromatin disruption in budding yeast. The yeast homologs of HMGB1

716 and SET, Hmo1 and Nap1 respectively, interact transiently with chromatin, but upon expression

717 of protein VII are recruited to the nucleosome to facilitate protein VII replacement of the linker

718 histone Hho1. b. Representative serial dilution assay for growth defects of induced and

719 uninduced W303 cells with protein VII expression plasmid in the indicated gene deletion strains.

720 Due to inherent slowed growth caused by deletion of HMO1, spots are shown after 4 days of

721 growth ( $\ddagger)$ for $h m o 1 \Delta$ and $h m 01 \Delta$ nap $1 \Delta$ strains. All other strains are shown after 3 days of 
722 growth. c. Quantification of serial dilution growth assays shown in (b). Relative growth was 723 measured as described in Figure 1. Growth density was quantified after 3 days growth for all 724 strains except the $h m 01 \Delta$ and $h m o 1 \Delta$ nap $1 \Delta$ strains which were imaged and quantified after 4 725 days $(\ddagger)$ to compensate for slowed growth caused by the deletion of HMO1. Using one-way 726 ANOVA with multiple comparisons against WT, we found that deletion of HMO1 and deletion of 727 both HMO1 and NAP1 in the same strain led to significantly greater relative growth intensity 728 ( $p=0.0009$ and $p=0.0090$, respectively). The average growth was slightly higher for the nap1 $\Delta$ 729 strain $(p=0.7085)$ and lower for the hho1 $\Delta$ strain $(p=0.0687)$ but not significantly. Errors bars 730 show SD. At least three biological replicates were quantified for each strain. d. Western blot 731 analysis of induced W303 gene deletion strains shown in (c) with protein VII expression 732 plasmid. Protein VII-HA is shown with Pgk1 loading control. e. Representative serial dilution 733 spot assay for growth of FY602 cells with and without induction of protein VII in the indicated 734 deletion strains. Growth after 4 days is shown for the $h m 01 \Delta$ and $h m o 1 \Delta$ nap $1 \Delta$ strains $(\ddagger)$ while 735 the other images were taken after 3 days growth. f. Quantification of serial dilution growth assay 736 in FY602 deletion strains when protein VII is expressed. Growth density was quantified after 3 737 days growth for all strains except the $h m o 1 \Delta$ and $h m 01 \Delta$ nap1 $\Delta$ strains which imaged and 738 quantified after 4 days ( $¥)$. Using one-way ANOVA with multiple comparisons compared to WT, 739 we found that deletion of HMO1, NAP1, or both genes together lead to a significant increase in 740 growth intensity ( $p=.0035, p<0.0001, p<0.0001$, respectively). The change in growth intensity 741 when HHO1 was deleted was not statistically significant $(p=0.9993)$. Error bars show SD. At 742 least four biological replicates were quantified for each strain. g. Western blot analysis of 743 induced FY602-based strains with protein VII expression plasmid. Protein VII-HA is shown 744 along with the Pgk1 loading control. 
a.

WT

b.

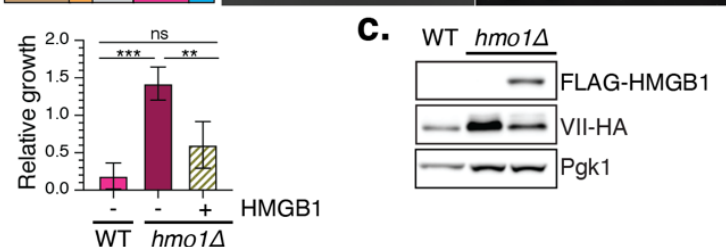

d.
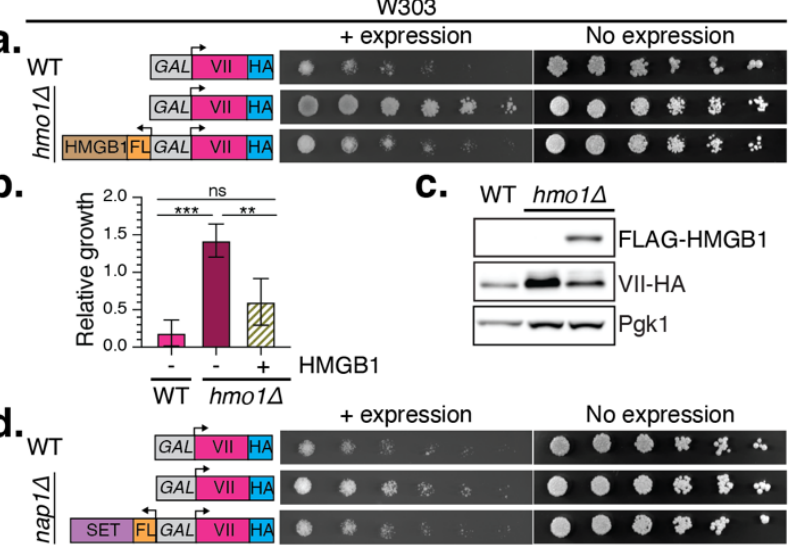

e.

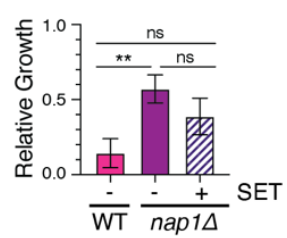

f.

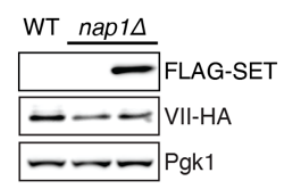

Figure 3. Replacing Hmo1 or Nap1 with homologous human factors exacerbates the protein VII growth defects in yeast. a. Serial dilution assay for protein VII growth defects in WT W303 and W303 hmo1s strain with and without co-expression of human HMGB1. Expression constructs are illustrated to the right. The bidirectional galactose-inducible promoter drives both protein VII and human HMGB1 transcription. Protein VII is HA-tagged and HMGB1 is FLAG-tagged. Cells without protein expression are shown after 3 days growth at $30^{\circ} \mathrm{C}$ while cells with protein expression are shown after 6 days growth at $30^{\circ} \mathrm{C}$ to compensate for slowed growth caused by protein VII and the non-preferred energy source, galactose. b. Quantification of serial dilution growth assay for strains shown in (a). As in (a), the cells with induced protein expression were quantified after 6 days and cell growth without protein expression was quantified after 3 days growth. A one-way ANOVA with multiple comparisons identified statistically significant differences in growth between WT and $h m 01 \Delta$ strains when VII is expressed $(p=0.0001)$. Likewise, growth with protein VII expression in hmo1s vs. VII coexpression with HMGB1 in the same strain was found to be significantly different $(p=0.0026)$.

763 The comparison of growth between WT + VII and $h m o 1 \Delta+$ VII + HMGB1 failed to pass the p-

764 value cut-off for significance $(p=0.068)$. Error bars represent SD for all growth quantification in

765 this figure. $\mathrm{N}=4$. c. Western blot analysis of WT W303 with protein VII, hmo1 $\Delta$ with VII, and $766 h m o 1 \Delta$ with protein VII and HMGB1 co-expression. HMGB1 is FLAG-tagged, protein VII is HA767 tagged, and Pgk1 is the loading control. d. Serial dilution assay for protein VII growth defects in 768 WT W303 and W303 nap1A strain with and without co-expression of human SET as described 
769 in (a). The co-expression construct that drives VII and SET from the same bidirectional 770 galactose promoter is illustrated at right. SET is FLAG-tagged, and protein VII is HA-tagged. e. 771 Quantification of serial dilution growth assay in WT and nap1 $\Delta$ when protein VII is expressed 772 alone or co-expressed with human SET as performed in (b). A one-way ANOVA with multiple 773 comparisons identified statistically significant differences in growth between WT and nap1 $\Delta$ 774 strains when VII is expressed $(p=0.0058)$. When SET was co-expressed with VII in nap1 1 , the 775 average growth intensity was reduced compared to VII expression alone in the same strain but 776 not significantly so $(p=0.0641)$. No significant difference was found between WT + VII and 777 nap1 $\Delta+\mathrm{VII}+\mathrm{SET}$ growth $(p=0.1604) . \mathrm{N}=3$. f. Western blot analysis of WT W303 with protein 778 VII, nap1 1 with VII, and nap1 1 with protein VII and SET co-expression. SET is FLAG-tagged, 779 protein VII is HA-tagged, and Pgk1 is the loading control. g. Serial dilution assay for growth with 780 protein VII expression in WT FY602 and FY602 hmo1 1 strain with and without co-expression of 781 human HMGB1 as in (a). h. Quantification of serial dilution growth assay in WT FY602 and 782 FY602 hmo1 1 when protein VII is expressed alone or co-expressed with human HMGB1 as in 783 (b). While the average growth intensity increased when HMO1 was deleted compared to WT, 784 the difference was not statistically significant (one-way ANOVA with multiple comparisons, $785 p=0.1132$ ). However, replacing Hmo1 with human HMGB1 resulted in a statistically significant reduction in growth $(p=0.0180)$. No significant difference in growth was detected when comparing WT and the HMGB1 replacement condition $(p=0.2982) . \mathrm{N}=3$. i. Western blot analysis of WT FY602 with VII, hmo1 1 with VII, and $h m 01 \Delta$ with VII and HMGB1 co-expression. Protein VII is HA-tagged, HMGB1 is FLAG-tagged, and Pgk1 is the loading control. j. Serial dilution growth assay for VII expression in WT FY602 and FY602 nap1 $\Delta$ strain with and without co-expression of human SET as described in (d). k. Quantification of serial dilution growth assay in WT FY602 and FY602 nap1 1 when protein VII is expressed alone or co-expressed with human SET as described in (b). Deletion of NAP1 rescued the protein VII growth 794 phenotype compared to WT (one-way ANOVA with multiple comparisons, $p=0.0003$ ). Adding 795 human SET to the NAP1 deletion strain reduced growth ( $\mathrm{p}=0.0240)$. Replacing Nap1 with SET 796 did not cause the same severity of growth defect as VII expression in WT $(p=0.0133)$. I. 797 Western blot analysis of WT FY602 with VII, nap1 $\Delta$ with VII, and nap $1 \Delta$ with VII and SET. VII is 798 HA-tagged, SET is FLAG-tagged, and Pgk1 is the loading control. 
a.

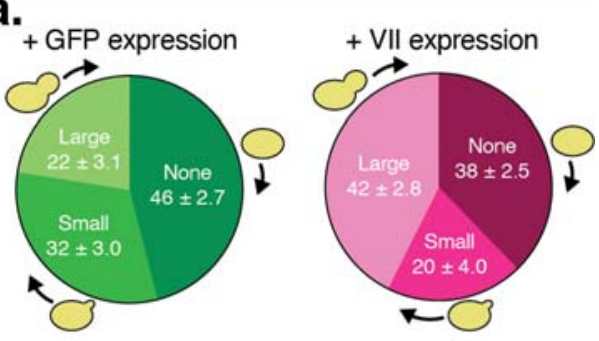

b.

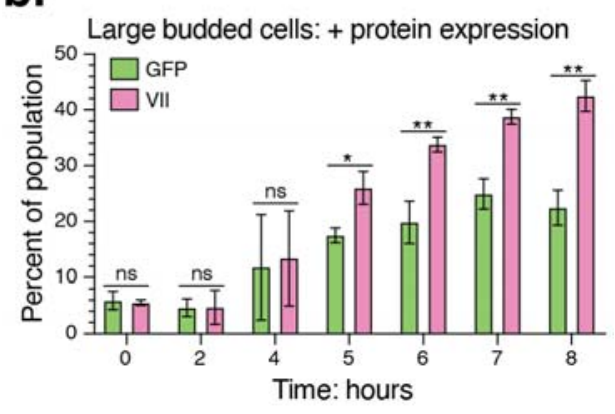

Figure 4. Protein VII dysregulates the yeast budding cycle. a. Budding analysis of WT W303 with GFP or protein VII expression mid-log phase growth after 8 hours growth in galactose media. Cells without a bud are in G1 while cells with a small bud are typically in S phase. A large bud roughly indicates G2/M before the cells completes mitosis and cytokinesis to produce a new cell. The percentage of the population with each bud type is shown along with SD. Using multiple unpaired t-tests (two-stage step-up method of Benjamini, Krieger and Yekutieli to control FDR), there was no significant difference in the proportion of unbudded $(p=0.0172)$ or small-budded cells $(p=0.0143)$. There was significant difference in the proportion of largebudded cells ( $p=0.0012$ ) upon expression of protein VII compared to GFP expressing cells.

$813 \mathrm{~N}=3$. b. The percentage of large budded cells in the population during protein VII expression 814 (pink) or GFP expression (green) in WT W303. At the beginning of the experiment, the cells in stationary phase (T0) did not express protein VII but were moved to galactose media to initiate protein expression and were grown to mid-log phase (T8). The $\mathrm{x}$-axis indicates time since

817 induction of protein expression and the start of the growth experiment. Unpaired $t$-test $P$-values 818 for each timed sample (T0-T8) from left to right on the x-axis are: $0.727079,0.962070$, $0.837029,0.010250,0.003903,0.001382$, and 0.001167 . $N=3$. 
a.

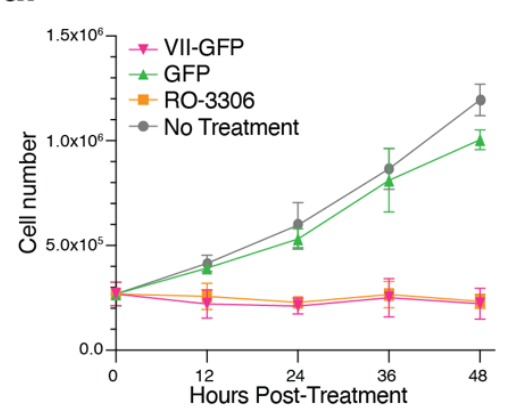

d.

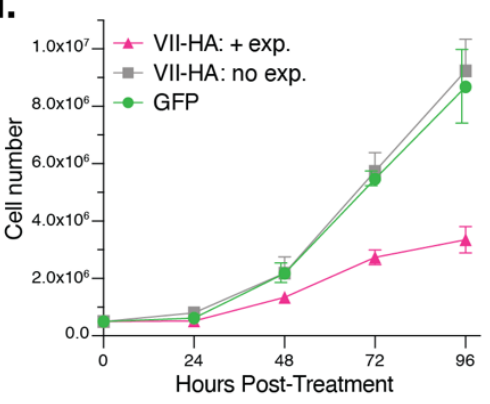

b.

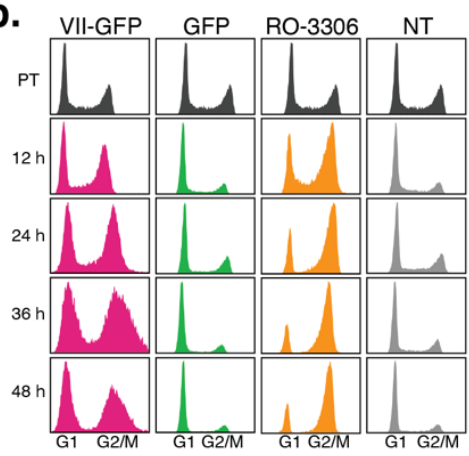

e.

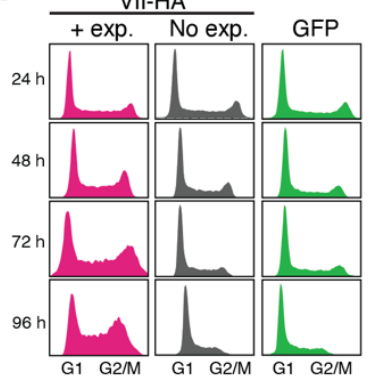

c.

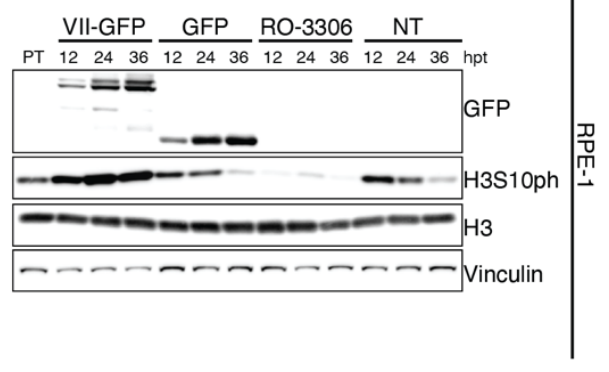

f.

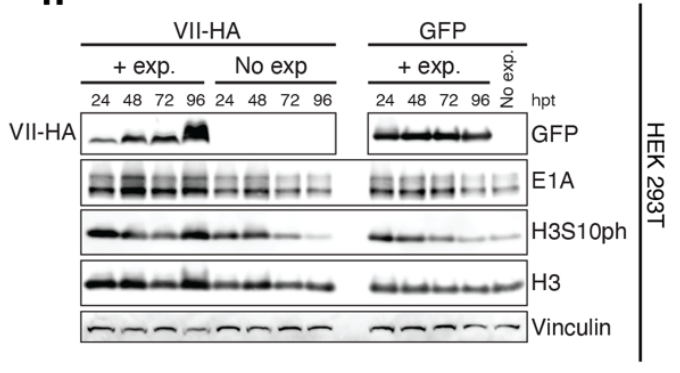

g.
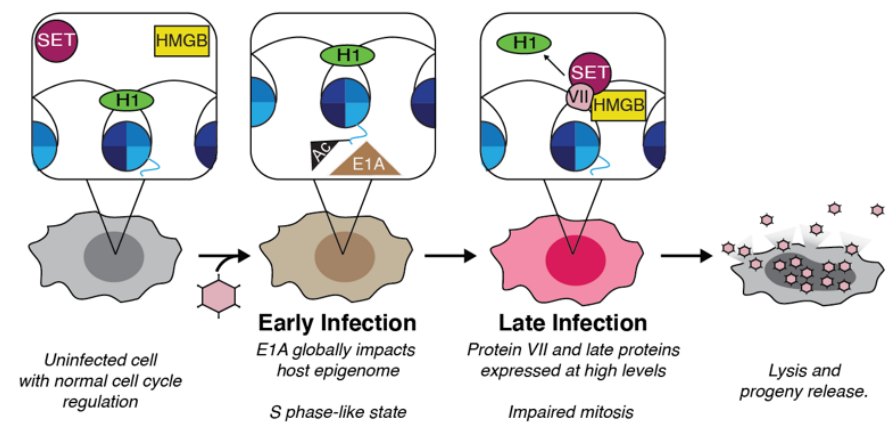

Figure 5. Protein VII blocks proliferation and impedes cell cycle progression in human cells. a. Diploid RPE-1 cell proliferation for 48 hours after treatment. The cells were transduced with recombinant adenovirus to express GFP-tagged protein VII or a GFP control. Protein VIIGFP recombinant adenovirus $\mathrm{MOI}=50$. GFP control recombinant adenovirus $\mathrm{MOI}=50.10 \mu \mathrm{M}$ RO-3306 CDK-1 inhibitor treatment is a control for inhibition of proliferation and G2 arrest. At 48 hours, there was a significant difference in the total number of protein VII-GFP expressing cells compared to the no treatment control $(p<0.0001$, one-way ANOVA with Tukey's multiple

832 comparisons test) and to the GFP control $(p<0.0001)$. There was no difference between the 833 cells expressing protein VII-GFP and RO-3306 treated cells $(p=0.9933)$. Error bars show 834 standard deviation. $\mathrm{N}=4$. b. Cell cycle analysis by flow cytometry of transduced RPE-1 cells 835 shown in (a). Cells were fixed and stained with propidium iodide to assess DNA content. DNA 836 content is plotted on the $x$-axis, with $G 1$ and $G 2 / M$ populations labelled, and the $y$-axis 
837 represents a histogram of the cell population. The y-axis is normalized to fill the entire axis 838 independently for each sample. Since nearly $100 \%$ of transduced cells were positive for GFP 839 expression, cells were not gated on GFP positive cells. The G1 and G2/M populations 840 correspond to cells with $2 \mathrm{~N}$ and $4 \mathrm{~N}$ DNA content respectively. c. Western blot analysis of 841 transduced RPE-1 cells shown in (a). Samples labeled "NT" are the no treatment control. "PT" 842 indicates the pre-treatment sample and "hpt" shows the time post-treatment in hours. The GFP 843 blot shows the relative protein VII-GFP and GFP expression. H3S10ph indicates mitotic 844 chromosomes. Total histone H3 and vinculin loading controls are also shown. d. HEK 293T 845 proliferation with and without protein VII-HA expression. The protein VII-HA + expression and 846 GFP cells were induced to express the target proteins under a doxycycline inducible promoter 847 for 96 hours. The protein VII-HA no expression cells were not treated with doxycycline. At 96 848 hours post-treatment, there were significantly fewer cells expressing protein VII-HA compared to 849 the GFP control and the untreated protein VII-HA cells (one-way ANOVA with multiple 850 comparisons; $p=0.0016$ and $p=0.0009$ respectively). There was no significant difference in cell 851 number between the GFP control and untreated protein VII-HA cells $(p=0.7841)$. Error bars 852 represent SD. $\mathrm{N}=3$. e. Flow cytometry cell cycle analysis of HEK 293T cells shown in (d) using 853 DNA content to assess cell cycle progression as described (b). Since more than $90 \%$ of the 854 doxycycline treated GFP cells were positive for GFP expression, the cells were not gated on positive expression. DNA content is shown on the x-axis and G1 and G2/M peaks are indicated although the ploidy of HEK 293T cells varies within the population of cells. The $y$-axis histogram 857 is normalized to fit the entire G1 peak for each sample. f. Western blot analysis of HEK 293T 858 cells shown in (d). HA- and GFP-probed blots indicate expression of target proteins in 859 doxycycline-treated samples. E1A indicates the presence of adenovirus early expressed E1A 860 protein which contributes to the transformation of HEK 293T cells. Multiple E1A isoforms are 861 resolved as multiple bands. H3S10ph indicates mitotic chromatin. Total histone H3 and vinculin 862 are loading controls. g. Model for protein VII on host chromatin during infection. An uninfected 863 cell has normal cell cycle regulation. Upon infection, the early-expressed adenovirus genes E1A 864 and E1B bypass cell cycle checkpoints and push the cell into an $S$ phase-like gene expression 865 program. E1A acts primarily through redistributing acetylation of histone H3K18 globally. Late 866 during infection, abundant protein VII recruits HMGB1 and SET to displace the linker histone 867 from host chromatin. Protein VII chromatin disruption impedes mitosis and therefore cytokinesis, 868 culminating in cell lysis and maximizing the release of viral progeny. 


\section{Supplemental Figures and Table}

872

\section{3}

874

875

876

877

878

879

880

881

882

883

884

885

886

887

888

889

890

891

892

893

894 a.

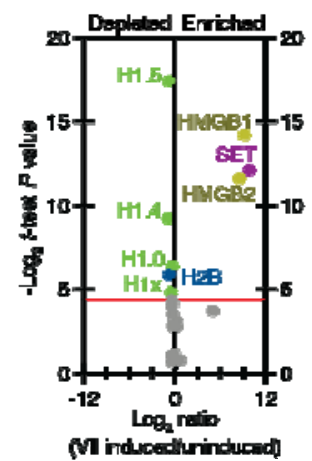

b.

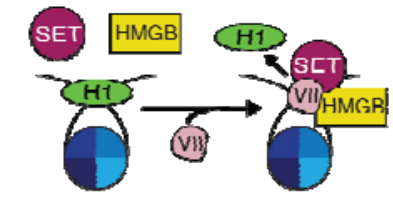

C.

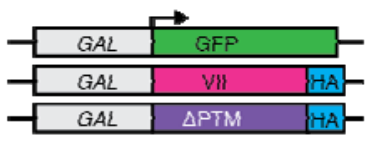

\section{Supplemental Figure 1. Binding of the histone-like adenovirus protein VII to chromatin} causes linker histone depletion.

a. Volcano plot of mass spectrometry analysis of salt-fractionated chromatin from cells with and without protein VII expression from our previously published study ${ }^{32}$. The data points correspond to protein SET (purple), HMGB proteins (gold), linker H1 (green), and all detected core canonical histones (blue, gray). The $y$-axis represents the $-\log _{2}$ statistical $p$-value and the $x$-axis represents the $\log _{2}$ protein fold-change between uninduced or protein VII-expressing cells (homoscedastic two-tailed t-test, $p<0.05$ ) indicating whether a protein was enriched or depleted from chromatin. The points shown in gray fall below the cutoff for statistical significance which is indicated by the red line. See Supplemental Table 1 for all protein identifiers and statistical values. b. Model for protein VII disruption of chromatin in human cells. Proteomics of protein VII-bound chromatin suggests its interaction with HMGB1 and SET facilitate protein VII invasion of chromatin and displacement of linker histone $\mathrm{H} 1$. In vitro, protein VII binds the nucleosome at a position similar to linker histones suggesting that $\mathrm{H} 1$ and VII compete for binding the nucleosome. c. Schematics of budding yeast expression constructs. The galactose promoter is truncated to drive transcription only in the direction indicated by the arrow. The plasmids code for either GFP, mature protein VII from WT adenovirus type 5, or protein VII with alanine substitutions at 5 sites of post-translational modification ( $\triangle \mathrm{PTM})$. VII and VII- $\triangle$ PTM genes are codon optimized for expression in $S$. cerevisiae and contain a $3 \times \mathrm{HA}$ epitope tag at the C-terminus. Each of these promoter and gene combinations is integrated into the same URA3-selectable plasmid backbone with a centromere to ensure high fidelity of transmission. 
a.

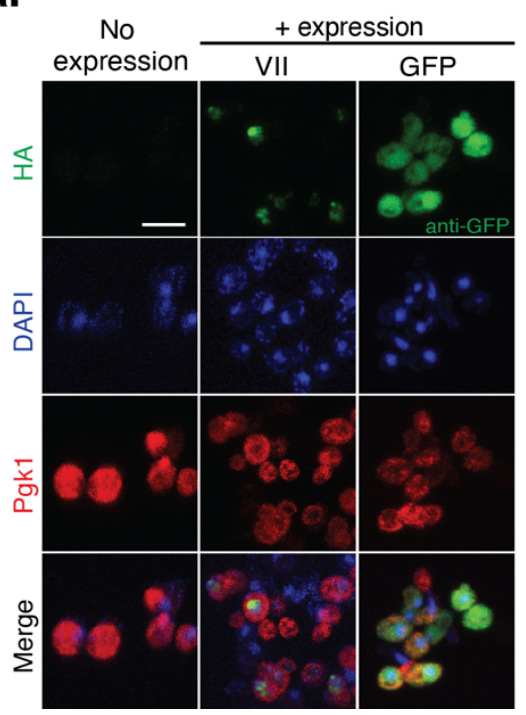

b.

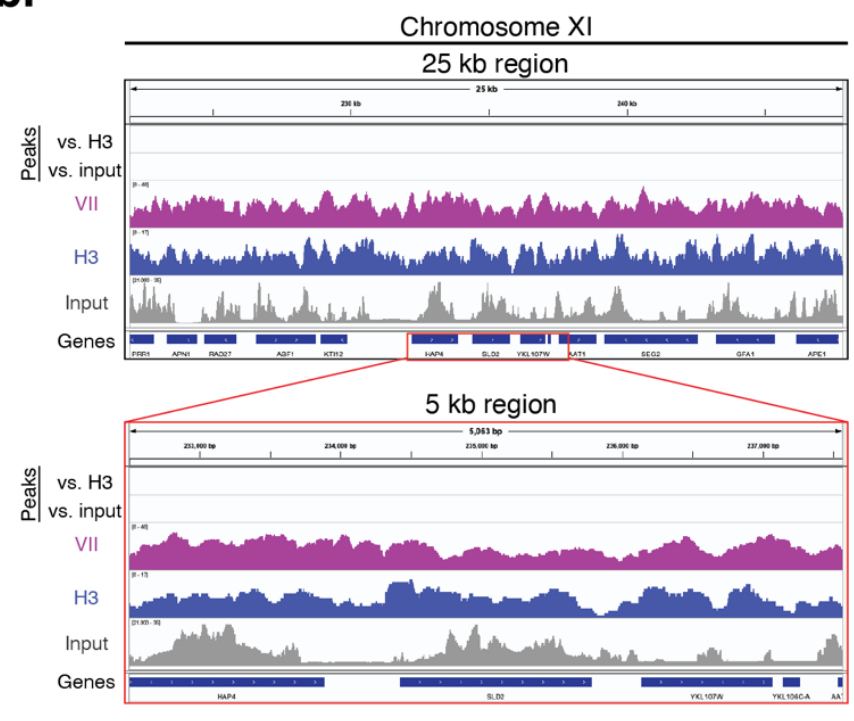

897 Supplemental Figure 2. Protein VII localizes to the nucleus and binds chromatin in yeast.

898 a. Immunofluorescence microscopy of WT FY602 cells with and without protein expression.

899 Pgk1 indicates the cytoplasm and DAPI stains the nucleus. Scale bar is $5 \mu \mathrm{m}$. b. Native ChIP900 seq of WT FY602 cells expressing protein VII. "Narrow peaks" called by MACS2 peak caller are 901 shown in the top two tracks along with IGV tracks for input, histone H3, and protein VII ChIP-seq 902 reads at one $25 \mathrm{~kb}$ locus on Chromosome XI. Red box indicates a smaller $5 \mathrm{~kb}$ region shown in 903 greater detail. All tracks were generated with MACS2 peak-caller using uniquely mapped reads.

904 Reads are normalized to counts per million. Tracks are automatically scaled for each 905 chromosome. Tracks from one experimental replicate are shown. ChIP-seq was performed in 906 duplicate. 

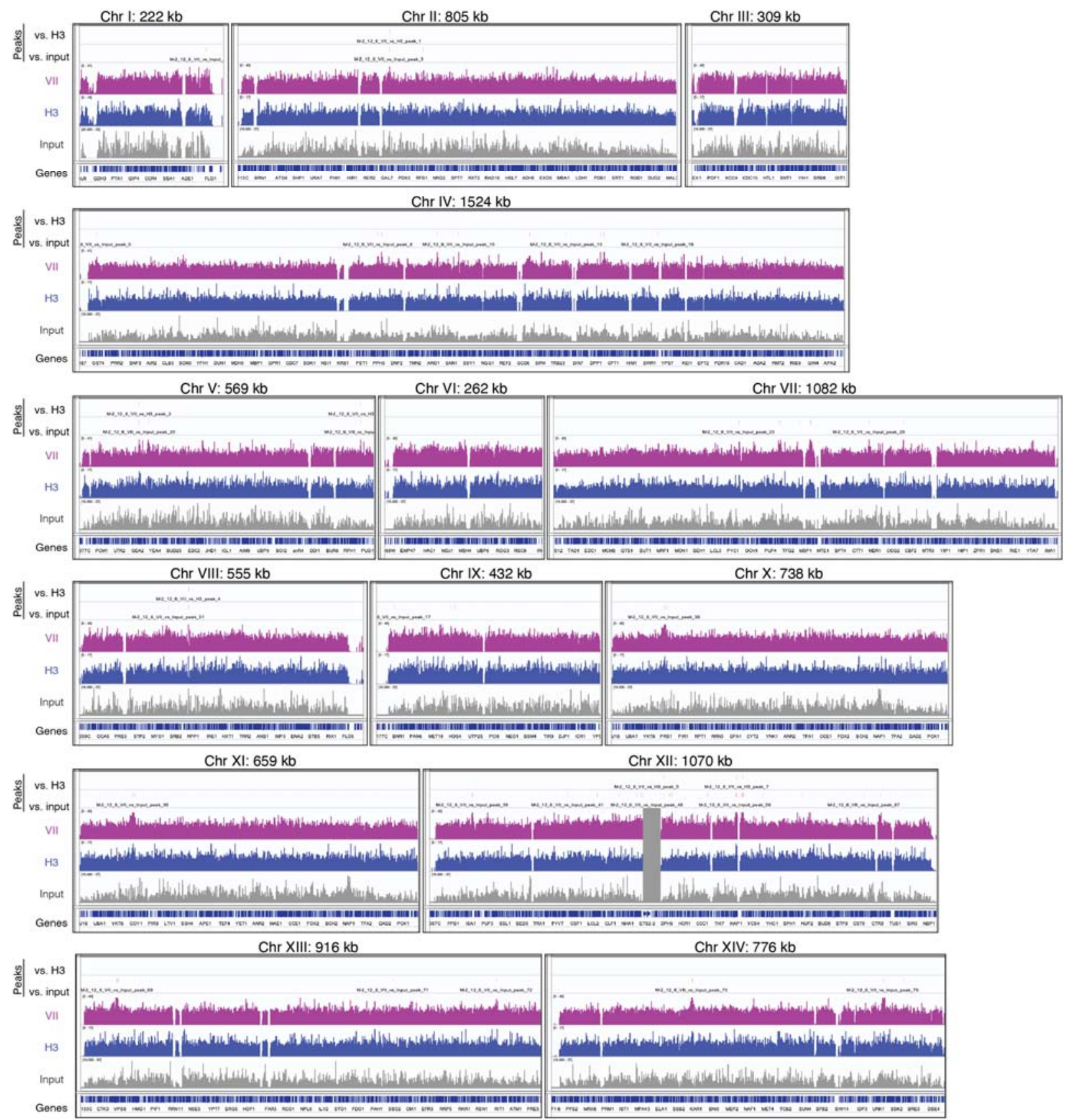

Chr XV: $1083 \mathrm{~kb}$

$\mathrm{Chr}$ XVI: $940 \mathrm{~kb}$
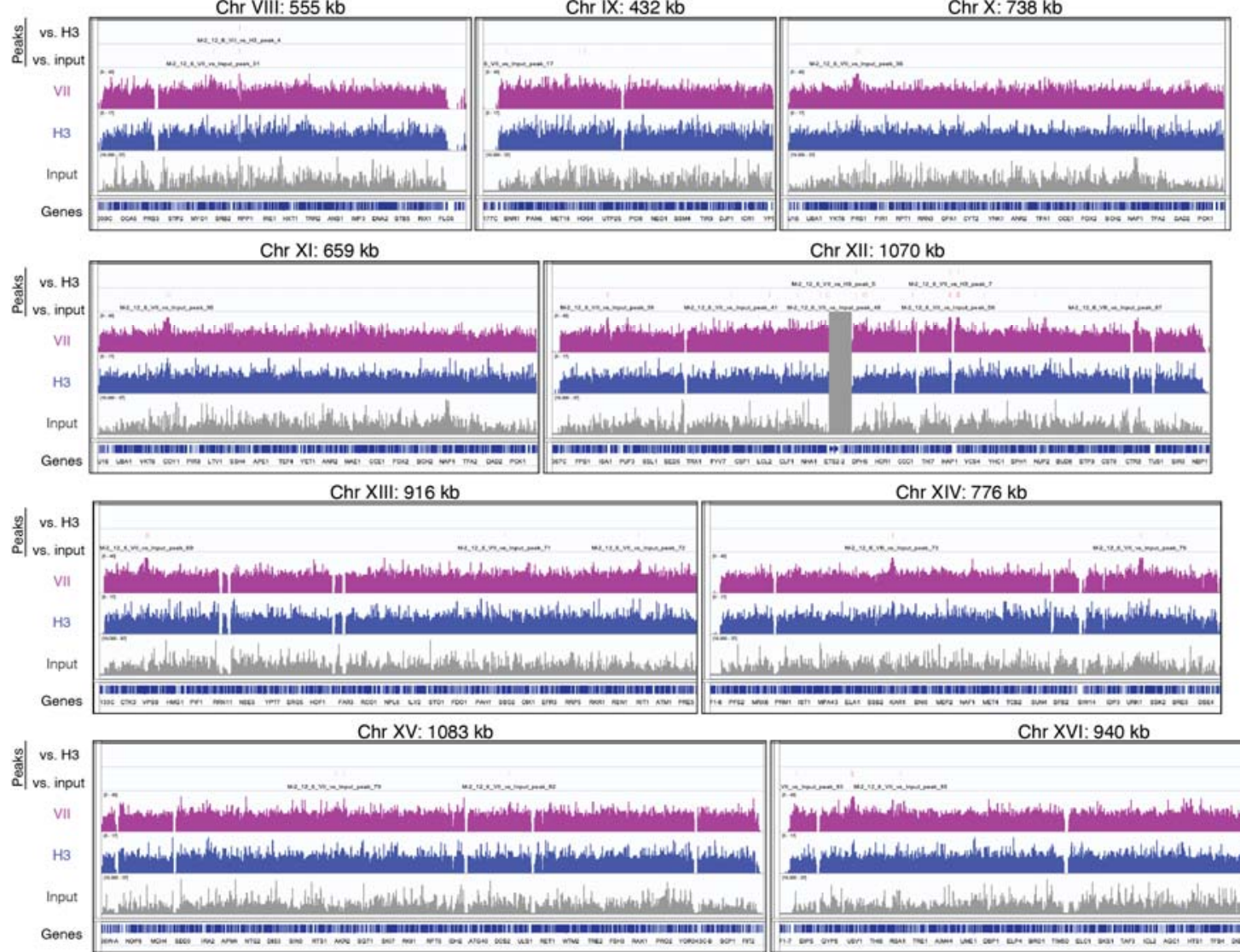

Chr XII: $1070 \mathrm{~kb}$

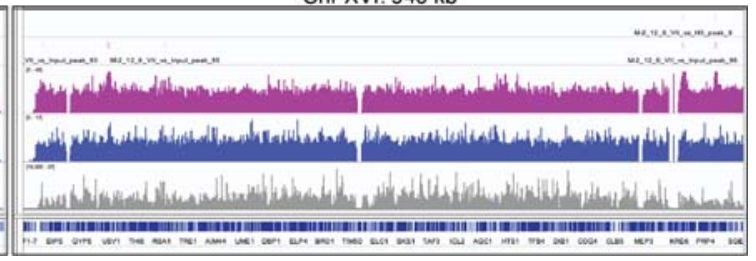

910 Supplemental Figure 3. Protein VII binds throughout yeast chromatin. "Narrow peaks"

911 called by MACS2 peak caller are shown along with IGV tracks for input, histone H3, and protein

912 VII ChIP-seq for all yeast chromosomes from WT FY602 as described in Supplemental Figure 
913 2b. Due to poor mapping, the reads at the rDNA locus (chromosome XII; $450-480 \mathrm{~kb}$ ) are 914 masked.

a.

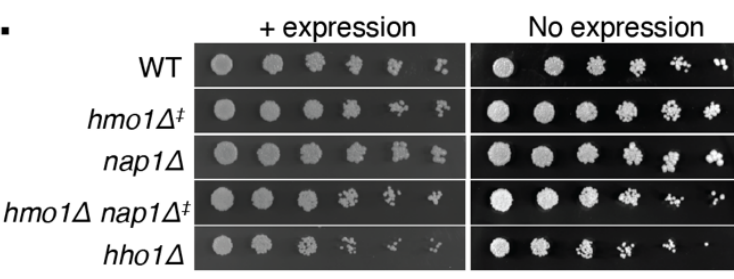

b.

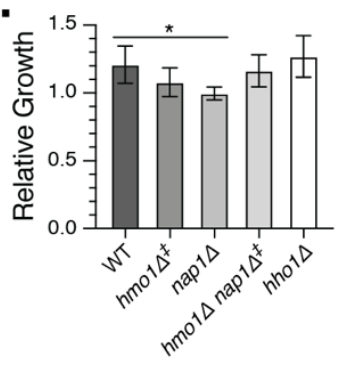

C.

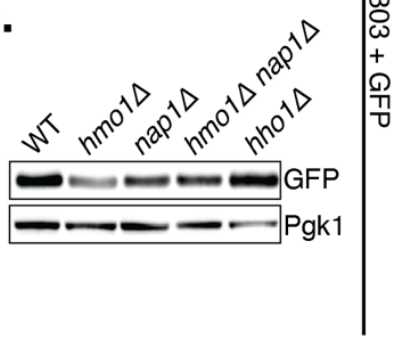

d.

+ expression

No expression

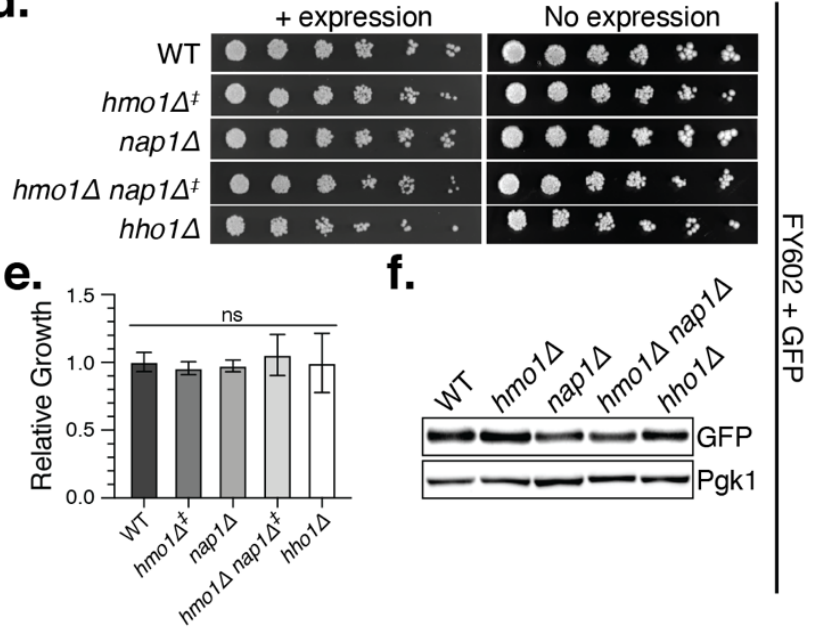

Supplemental Figure 4. Loss of Hmo1 or Nap1 has no significant impact on growth when GFP is expressed. a. Representative serial dilution assay for growth defects of induced and repressed W303 cells with GFP expression plasmid in the indicated gene deletion strains as in Figure 2b. b. Quantification of serial dilution growth assay in W303 gene deletion strains during GFP expression. Relative growth was quantified as in Figure 2c for at least three biological

921 replicates. No statistical significance was found when WT growth was compared to each 922 deletion strain except for the nap1 $\Delta$ strain, although the $p$-value barely exceeded the cut-off for 923 significance (One-way ANOVA with multiple comparisons vs. WT; $p=0.0443$ ). $P$-values for 924 remaining comparisons to WT: $h m 01 \Delta=0.3213$, nap1 $\Delta=0.0443$, $h$ mo1 $\Delta$ nap $1 \Delta=0.9583$, $925 h h 01 \Delta=0.8666$. Error bars represent SD. c. Western blot analysis of induced W303 gene 926 deletion strains with GFP expression plasmid and Pgk1 as a loading control. d. Representative 927 serial dilution assay for growth defects in induced and repressed FY602 cells with GFP 
928 expression plasmid in the indicated gene deletion strains as described in Figure $2 b$. e. 929 Quantification of serial dilution growth assay in FY602-based deletion strains when GFP is 930 expressed as described in Figure 2c. No statistical significance was determined among the 931 deletion strains when compared to WT growth using one-way ANOVA with multiple 932 comparisons. $P$-values for comparisons vs. WT: hmo1 $=0.8159$, nap1 $\Delta=0.9723$, $933 h m 01 \Delta$ nap $1 \Delta=0.8202, h h o 1 \Delta=0.9998$. Error bars depict SD. At least four biological replicates 934 were quantified for each strain. f. Western blot analysis of induced WT FY602 cells and related 935 deletion strains with GFP expression plasmid and Pgk1 shown as the loading control. 
a.

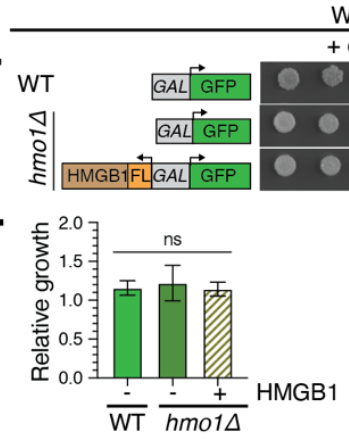

d.
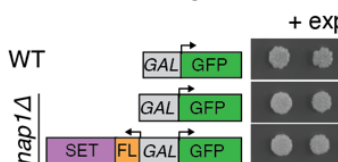

e.

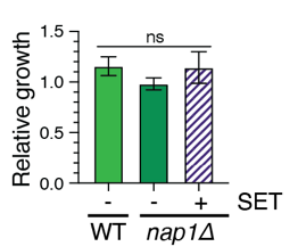

$\mathrm{N} 303+\mathrm{GFP}$

expression No expression $\rightarrow+00+4 *$

c.

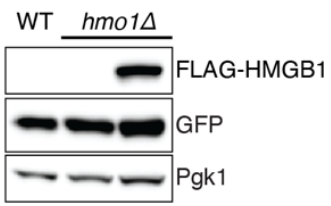

expression No expression $0 * *$ *

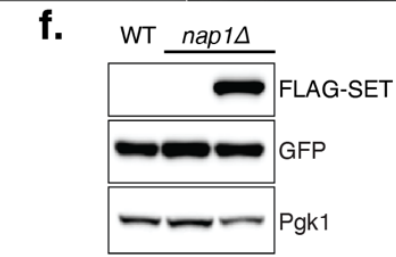

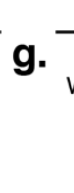

h.
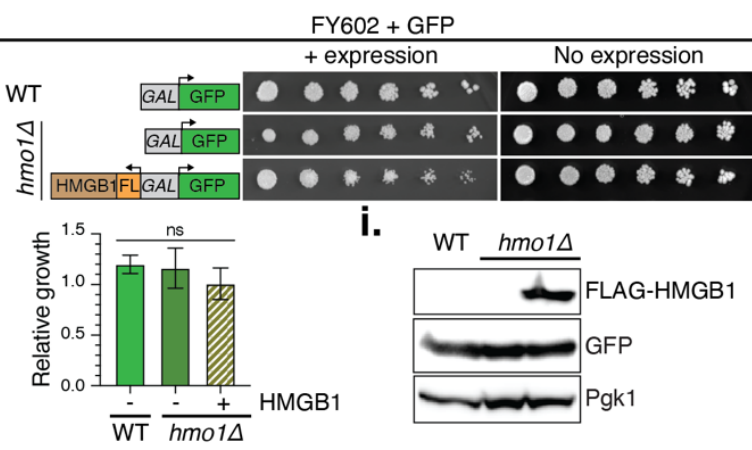

WT $h$ mo1s

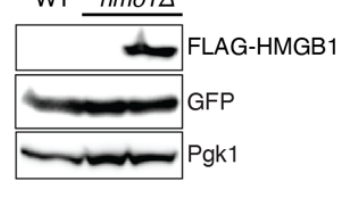

j.

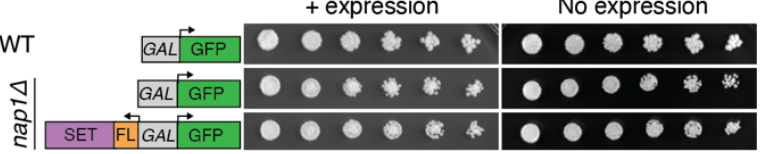

k.

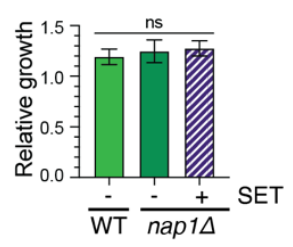

I. WT nap14

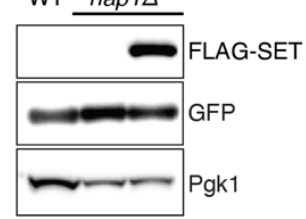

Supplemental Figure 5. Replacing Hmo1 or Nap1 with homologous human factors has no significant impact on growth when GFP is expressed. a. Serial dilution spot assay for GFP control in WT W303 and W303 hmo1 strain with and without co-expression of human HMGB1.

943 Expression constructs illustrated to the right show the bidirectional galactose-inducible promoter 944 that drives GFP and human HMGB1 transcription. Cells without protein expression are shown 945 after 3 days growth at $30^{\circ} \mathrm{C}$ while cells with protein expression are shown after 5 days growth at $94630^{\circ} \mathrm{C}$ to compensate for slowed growth on the non-preferred energy source, galactose. b.

947 Quantification of serial dilution growth assay of strains shown in (a). Growth was quantified after

9485 days growth with protein expression and compared to growth after 3 days growth without 949 protein expression. A one-way ANOVA with multiple comparisons showed no significant 950 difference in growth among the three different strains and co-expression constructs (WT + GFP 951 vs. $h m o 1 \Delta+$ GFP $p=0.8426$; WT + GFP vs. $h m o 1 \Delta+$ GFP + HMGB1 $p=0.9922, h m o 1 \Delta+$ GFP 952 vs. $h m o 1 \Delta+$ GFP + HMGB1 $p=0.8205)$. Error bars represent SD for all growth quantification in 953 this figure. $\mathrm{N}=3$. c. Western blot analysis of strains shown in (a). FLAG-HMGB1, GFP, and 954 Pgk1 loading control are shown. d. Serial dilution growth assay for GFP control in WT W303 955 and W303 nap14 strain with and without co-expression of human SET. Bidirectional galactose 956 promoter and target genes are illustrated at right. Images depicting growth were selected as 957 described in (a). e. Quantification of serial dilution growth assay in WT W303 and W303 nap14 958 when GFP is expressed alone or co-expressed with human SET as described in (b). A one-way 
ANOVA with multiple comparisons failed to detect statistically significance differences in growth among these strains (WT + GFP vs. nap1 + GFP $p=0.1181 ;$ nap1 $\Delta+$ GFP + SET $p=0.9832$,

$961 \mathrm{WT}+\mathrm{GFP}$ vs. nap1 $\Delta+\mathrm{GFP}+\mathrm{SET} p=0.1536) . \mathrm{N}=3$. f. Western blot analysis from strains

962 shown in (d). GFP and FLAG-SET blots are shown with Pgk1 loading control. g. Serial dilution

963 growth assay for GFP expression in WT FY602 and FY602 hmo1 1 strain with and without co-

964 expression of human HMGB1 as described in (a). h. Quantification of serial dilution growth

965 assay in WT W303 and W303 hmo1 when GFP is expressed alone or co-expressed with

966 human HMGB1 as described in (b). There was no significant difference in growth among the

967 three strains (one-way ANOVA with multiple comparisons; WT + GFP vs. $h m 01 \Delta+$ GFP

$968 p=0.9517$; WT + GFP vs. $h m 01 \Delta+\mathrm{GFP}+\mathrm{HMGB} 1 p=0.3470, h m o 1 \Delta+\mathrm{GFP}$ vs. $h m o 1 \Delta+\mathrm{GFP}$

$969+$ HMGB1 $p=0.4853) . \mathrm{N}=3$. i. Western blot analysis of WT FY602 with GFP, $h m 01 \Delta$ with GFP,

970 and $h m 01 \Delta$ with GFP and HMGB1 co-expression. FLAG-HMGB1, GFP, and Pgk1 loading

971 control blots are shown. j. Serial dilution growth assay for GFP in WT FY602 and FY602 nap1D

972 strain with and without co-expression of human SET as described in (d). k. Quantification of

973 serial dilution growth assay in WT W303 and W303 nap1 $1 \Delta$ wen GFP is expressed alone or co-

974 expressed with human SET as in (b). There was no significant difference in growth among the

975 three strains (one-way ANOVA with multiple comparisons; WT + GFP vs. nap1 + GFP

$976 p=0.7047$; WT + GFP vs. nap $1 \Delta+$ GFP + SET $p=0.4743$, nap1 $\Delta+$ GFP vs. nap $1 \Delta+$ GFP +

977 SET $p=0.9222$ ). $N=3$. I. Western blot analysis of strains shown in (j). FLAG-SET, GFP, and

978 Pgk1 loading control blots are shown.

979

980 
a.
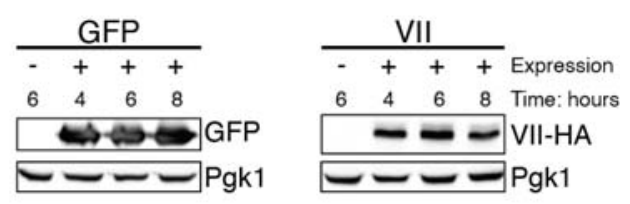

b.
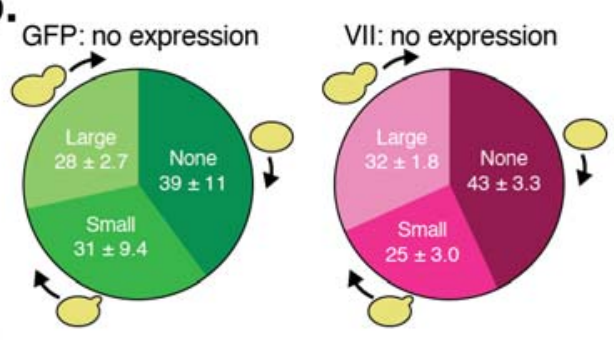

c.

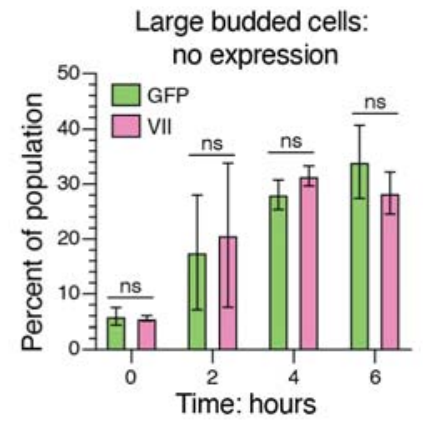

\section{Supplemental Figure 6. The accumulation of large-budded cells requires protein VII} expression. a. Western blot analysis of timed samples collected during budding analysis of WT W303. Time indicates total time in hours that the cells grew in the no expression (dextrose) or + expression (galactose) condition. GFP, VII-HA, and Pgk1 loading control blots are shown. b. Budding analysis of strains shown in Figure 4 without induction of protein expression and after 4 hours asynchronous growth in dextrose media. The percentage of the population with each bud type is shown along with SD. Multiple unpaired t-tests failed to identify a statistically significant difference in the proportion of cells in each budding class between the two strains $(p=0.6284$ for unbudded cells, $p=0.3329$ for small-budded cells, and $p=0.1445$ for large-budded cells). $N=3$ in WT W303. c. The percentage of large-budded cells in the population during asynchronous growth without induction of GFP (green) or protein VII (pink) expression. At the beginning of the 994 experiment, the cells in stationary phase (T0) were moved to dextrose media to repress protein 995 expression and were grown to mid-log phase (T6). The s-axis indicates the time since the 996 addition of dextrose and the start of the growth experiment. Unpaired $t$-test $P$-values for each 997 timed sample in order as shown on the graph from T0 to T6 are: $0.7271,0.7601,0.1445$, and $998 \quad 0.2701 . \mathrm{N}=3$ in WT W303. 
a.

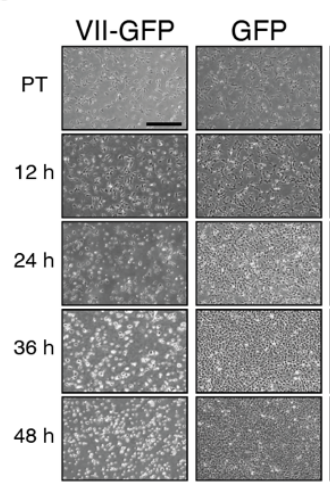

d.

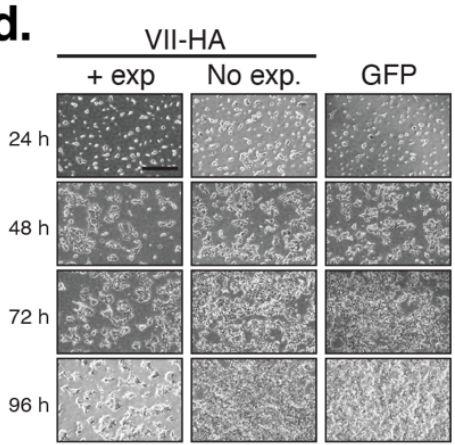

b.

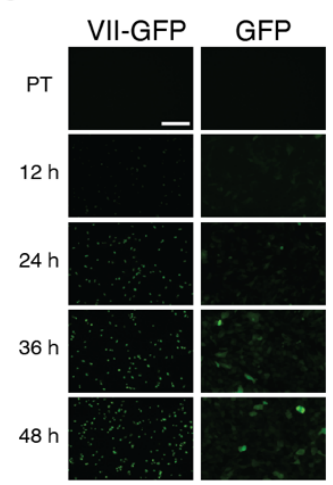

e.
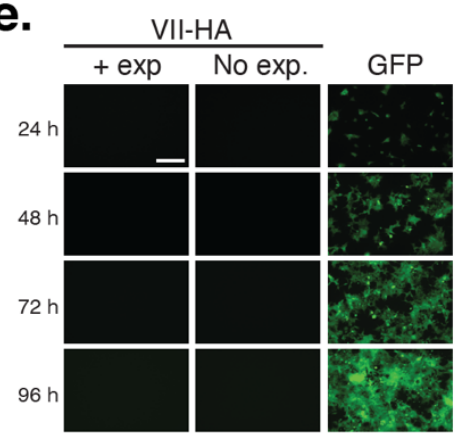

C.

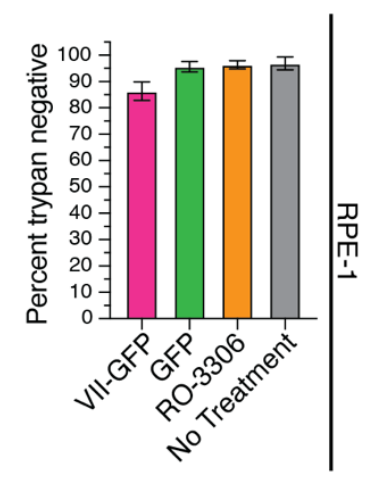

f.

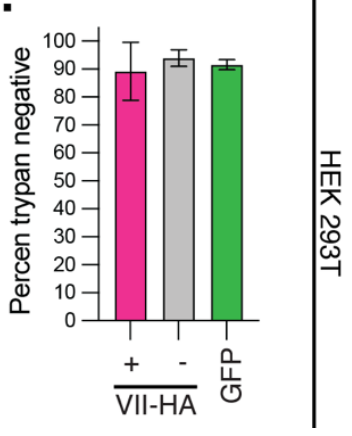

Supplemental Figure 7. Non-proliferative cells that express protein VII are metabolically active. a. Brightfield microscopy images of diploid RPE-1 cells show the loss of proliferation in cells transduced to express protein VII-GFP, or treated with RO-3306, compared to the no treatment and GFP controls. $5 x$ magnification. Scale bar is $500 \mu \mathrm{m}$.

b. Fluorescent microscopy images of RPE-1 cells that express either protein VII-GFP or GFP. 10x magnification. Scale bar

c. Percentage of RPE-1 cells that are negative for trypan blue exclusion stain 48 1008 hours post-treatment. Errors bars are SD. $\mathrm{N}=4$. d. Brightfield microscopy images of HEK 293T cells show the loss of proliferation in cells expressing protein VII-HA but not the untreated control or cells expressing GFP. 5x magnification. Scale bar is $500 \mu \mathrm{m}$. e. Green fluorescent 1011 microscopy images of HEK 293T cell lines with and without doxycycline induction of protein 1012 expression. 10x magnification. Scale bar is $200 \mu \mathrm{m}$. f. Percentage of HEK 293T cells negative 1013 for trypan blue exclusion stain 96 hours post-treatment. "+/-" indicates doxycycline treatment 1014 status of VII-HA cells. The GFP cells are induced with doxycycline. Errors bars are SD. N = 3. 


\begin{tabular}{|c|c|c|c|}
\hline Protein Name & Gene Names & $\begin{array}{l}t \text {-test } P \text { - } \\
\text { value }\end{array}$ & $\begin{array}{l}\text { Log2 fold change: } \\
\text { VII induced/uninduced }\end{array}$ \\
\hline Histone H1.5 & HIST1H1B & $5.69851 \mathrm{E}-06$ & -0.757738748 \\
\hline $\begin{array}{l}\text { High mobility group protein } \\
\text { B1;Putative high mobility group } \\
\text { protein B1-like } 1\end{array}$ & HMGB1; HMGB1P1 & 5.38059E-05 & 9.17634819 \\
\hline Protein SET & SET & 0.000229685 & 9.848560151 \\
\hline High mobility group protein B2 & HMGB2 & 0.000322399 & 8.559909323 \\
\hline Histone H1.4 & HIST1H1E & 0.001652512 & -0.903455652 \\
\hline $\begin{array}{l}\text { Histone } \mathrm{H} 1.0 ; \text { Histone } \mathrm{H} 1.0, \mathrm{~N}- \\
\text { terminally processed }\end{array}$ & H1F0 & 0.012110662 & -0.359847618 \\
\hline Histone H2B type 3-B & HIST3H2BB & 0.017135066 & -0.68225997 \\
\hline Histone $\mathrm{H} 1 \mathrm{x}$ & H1FX & 0.035053385 & -0.495869309 \\
\hline $\begin{array}{l}\text { Histone } \mathrm{H} 2 \mathrm{~A} \text { type } 1-\mathrm{C} ; \text { Histone } \mathrm{H} 2 \mathrm{~A} \\
\text { type } 3 \text {;Histone } \mathrm{H} 2 \mathrm{~A} \text {; Histone } \mathrm{H} 2 \mathrm{~A} \\
\text { type 1-B/E }\end{array}$ & $\begin{array}{l}\text { HIST1H2AC; HIST3H2A; } \\
\text { HIST1H2AB }\end{array}$ & 0.05001239 & -0.398885931 \\
\hline Histone H1.2 & HIST1H1C & 0.060260505 & -0.410806769 \\
\hline Histone H3;Histone H3.3 & H3F3B; H3F3A & 0.076305074 & 5.001910361 \\
\hline Histone H4 & $\begin{array}{l}\text { HIST1H4A; HIST1H4L; } \\
\text { HIST1H4H }\end{array}$ & 0.086418311 & -0.304855891 \\
\hline $\begin{array}{l}\text { Histone H2A.V;Histone } \\
\text { H2A.Z;Histone H2A }\end{array}$ & H2AFV; H2AFZ & 0.119555025 & 0.216370185 \\
\hline $\begin{array}{l}\text { Histone } \mathrm{H} 2 \mathrm{~A} \text { type } 2-\mathrm{C} \text {; Histone } \mathrm{H} 2 \mathrm{~A} \\
\text { type } 2-\mathrm{A}\end{array}$ & HIST2H2AC; HIST2H2AA3 & 0.140693297 & -0.2801282 \\
\hline $\begin{array}{l}\text { Histone H2B type 1-M;Histone H2B } \\
\text { type 1-N;Histone H2B type 1-H; } \\
\text { Histone H2B type 2-F;Histone H2B } \\
\text { type 1-C/E/F/G/l;Histone H2B type } \\
\text { 1-D;Histone H2B;Histone H2B type } \\
\text { 1-K;Histone H2B type F-S }\end{array}$ & $\begin{array}{l}\text { HIST1H2BM; HIST1H2BN; } \\
\text { HIST1H2BH; HIST2H2BF; } \\
\text { HIST1H2BC; HIST1H2BD; } \\
\text { HIST1H2BI; HIST1H2BK; } \\
\text { H2BFS }\end{array}$ & 0.144611424 & 0.26332697 \\
\hline $\begin{array}{l}\text { Histone H2A.x; Histone H2A type 1- } \\
\text { A }\end{array}$ & H2AFX; HIST1H2AA & 0.146404666 & -0.185106615 \\
\hline $\begin{array}{l}\text { Histone H2B type 2-E;Histone H2B } \\
\text { type 1-B;Histone H2B type 1- } \\
\text { O;Histone H2B type 1-J }\end{array}$ & $\begin{array}{l}\text { HIST2H2BE; HIST1H2BB; } \\
\text { HIST1H2BO; HIST1H2BJ }\end{array}$ & 0.456113639 & -0.103554106 \\
\hline $\begin{array}{l}\text { Histone H2B type 1-L;Histone H2B } \\
\text { type 1-A }\end{array}$ & HIST1H2BL; HIST1H2BA & 0.479413333 & -0.409269021 \\
\hline Histone H3.2 & HIST2H3A & 0.574677306 & -0.226071755 \\
\hline Histone H3.1; Histone H3.1t & HIST1H3A; HIST3H3 & 0.584639377 & 0.694148161 \\
\hline Histone H1.3 & HIST1H1D & 0.633380729 & -0.344162535 \\
\hline
\end{tabular}

\section{Supplemental Table 1.}

1019 A subset of protein and gene identifiers as well as statistical and enrichment scores for 1020 proteomics of salt-fractionated chromatin from cells with and without protein VII expression as 1021 previously published ${ }^{32}$. Here, the $t$-test $p$-values and enrichment scores corresponding to protein 1022 SET, HMGB proteins, linker H1 types, and the core canonical histones are shown. The values 1023 shown in gray-filled boxes were not statistically significant $(p<0.005)$. 
References cited:

1. Horwitz, G. A., Zhang, K., McBrian, M. A., Grunstein, M., Kurdistani, S. K. \& Berk, A. J. Adenovirus Small e1a Alters Global Patterns of Histone Modification. Science 321, 1084-1085 (2008).

1030 2. Ferrari, R., Pellegrini, M., Horwitz, G. A., Xie, W., Berk, A. J. \& Kurdistani, S. K. Epigenetic 1031 Reprogramming by Adenovirus ela. Science 321, 1086-1088 (2008).

1032 3. Fonseca, G. J., Thillainadesan, G., Yousef, A. F., Ablack, J. N., Mossman, K. L., Torchia, J. \& 1033 Mymryk, J. S. Adenovirus Evasion of Interferon-Mediated Innate Immunity by Direct 1034 Antagonism of a Cellular Histone Posttranslational Modification. Cell Host Microbe 11, 5971035606 (2012).

1036 4. Ferrari, R., Su, T., Li, B., Bonora, G., Oberai, A., Chan, Y., Sasidharan, R., Berk, A. J., 1037 Pellegrini, M. \& Kurdistani, S. K. Reorganization of the host epigenome by a viral oncogene. 1038 Genome Res 22, 1212-1221 (2012).

1039 5. Marazzi, I., Ho, J. S. Y., Kim, J., Manicassamy, B., Dewell, S., Albrecht, R. A., Seibert, C. 1040 W., Schaefer, U., Jeffrey, K. L., Prinjha, R. K., Lee, K., García-Sastre, A., Roeder, R. G. \& 1041 Tarakhovsky, A. Suppression of the antiviral response by an influenza histone mimic. Nature 1042 483, 428-433 (2012).

1043 6. Fonseca, G. J., Cohen, M. J., Nichols, A. C., Barrett, J. W. \& Mymryk, J. S. Viral Retasking of 1044 hBre1/RNF20 to Recruit hPaf1 for Transcriptional Activation. Plos Pathog 9, e1003411 (2013). Domain Regulates H3 Acetylation Affecting Varied Steps in Transcription at Different Viral Promoters. J Virol 92, e00805-18 (2018). perspectives and challenges. Nucleic Acids Res 41, 9593-9609 (2013).

9. Hergeth, S. P. \& Schneider, R. The H1 linker histones: multifunctional proteins beyond the nucleosomal core particle. Embo Rep 16, 1439-1453 (2015). regulating chromatin structure and function. Nat Rev Mol Cell Bio 19, 192-206 (2018). 
13. Štros, M. HMGB proteins: Interactions with DNA and chromatin. Biochimica Et Biophysica Acta Bba - Gene Regul Mech 1799, 101-113 (2010). Z., Sun, X., Wang, H., Wang, Q., Tsung, A., Billiar, T. R., Zeh, H. J., Lotze, M. T. \& Tang, D. HMGB1 in health and disease. Mol Aspects Med 40, 1-116 (2014). in chromatin. Biochimica Et Biophysica Acta Bba - Gene Regul Mech 1859, 462-467 (2016). 267, 552-554 (1977). adenovirions. Nucleic Acids Research 11, 441-460 (1983).

18. Vayda, M. E. \& Flint, S. J. Isolation and characterization of adenovirus core nucleoprotein subunits. Journal of Virology 61, 3335-3339 (1987).

1072 19. Haruki, H., Gyurcsik, B., Okuwaki, M. \& Nagata, K. Ternary complex formation between 1073 DNA $\square$ adenovirus core protein VII and TAF $\square \mathrm{I} / \mathrm{S}$ SET, an acidic molecular chaperone. Febs Lett $1074555,521-527(2003)$.

1075 20. Xue, Y., Johnson, J. S., Ornelles, D. A., Lieberman, J. \& Engel, D. A. Adenovirus Protein VII Functions throughout Early Phase and Interacts with Cellular Proteins SET and pp32. J Virol 1077 79, 2474-2483 (2005). precursor of adenovirus major core protein VII to DNA and template activating factor I: implication for the mechanism of remodeling of the adenovirus chromatin. Biochemistry 45, $1081303-313$ (2006).

22. Okuwaki, M. \& Nagata, K. Template activating factor-I remodels the chromatin structure and stimulates transcription from the chromatin template. The Journal of biological chemistry $\mathbf{2 7 3}$, 1084 34511-34518 (1998). Activating Factor I/SET in Transcription of Adenovirus Early Genes as a Positive-Acting Factor. J Virol 80, 794-801 (2006). 
26. Frisch, S. M. \& Mymryk, J. S. Adenovirus-5 E1A: paradox and paradigm. Nat Rev Mol Cell Bio 3, 441-452 (2002).

27. Ben-Israel, H. \& Kleinberger, T. Adenovirus and cell cycle control. Frontiers in bioscience $\square$ : a journal and virtual library 7, d1369-95 (2002).

28. Knipe, D. M. \& Howley, P. Fields Virology. (Lippincott Williams \& Wilkins, 2013).

29. Montell, C., Courtois, G., Eng, C. \& Berk, A. Complete transformation by adenovirus 2 requires both E1A proteins. Cell 36, 951-961 (1984).

30. BAYLEY, S. \& MYMRYK, J. ADENOVIRUS E1A PROTEINS AND TRANSFORMATION (REVIEW). Int J Oncol 5, 425-44 (1994).

31. King, C. R., Zhang, A., Tessier, T. M., Gameiro, S. F. \& Mymryk, J. S. Hacking the Cell: Network Intrusion and Exploitation by Adenovirus E1A. Mbio 9, e00390-18 (2018).

32. Avgousti, D. C., Herrmann, C., Kulej, K., Pancholi, N. J., Sekulic, N., Petrescu, J., Molden, R. C., Blumenthal, D., Paris, A. J., Reyes, E. D., Ostapchuk, P., Hearing, P., Seeholzer, S. H., Worthen, G. S., Black, B. E., Garcia, B. A. \& Weitzman, M. D. A core viral protein binds host nucleosomes to sequester immune danger signals. Nature 535, 173-177 (2016).

33. Avgousti, D. C., Fera, A. N. D., Otter, C. J., Herrmann, C., Pancholi, N. J. \& Weitzman, M. D. Adenovirus Core Protein VII Downregulates the DNA Damage Response on the Host Genome. J Virol 91, e01089-17 (2017).

34. Herrmann, C., Avgousti, D. C. \& Weitzman, M. D. Differential Salt Fractionation of Nuclei to Analyze Chromatin-associated Proteins from Cultured Mammalian Cells. Bio-protocol 7, (2017).

35. Adachi, Y., Pavlakis, G. N. \& Copeland, T. D. Identification and characterization of SET, a nuclear phosphoprotein encoded by the translocation break point in acute undifferentiated leukemia. The Journal of biological chemistry 269, 2258-2262 (1994).

36. Kato, K., Okuwaki, M. \& Nagata, K. Role of Template Activating Factor-I as a chaperone in linker histone dynamics. J Cell Sci 124, 3254-3265 (2011).

37. Kajitani, K., Kato, K. \& Nagata, K. Histone H1 chaperone activity of TAF $\square$ I is regulated by its subtype $\square$ dependent intramolecular interaction. Genes Cells 22, 334-347 (2017).

38. Willcockson, M. A., Healton, S. E., Weiss, C. N., Bartholdy, B. A., Botbol, Y., Mishra, L. N., Sidhwani, D. S., Wilson, T. J., Pinto, H. B., Maron, M. I., Skalina, K. A., Toro, L. N., Zhao, J., Lee, C.-H., Hou, H., Yusufova, N., Meydan, C., Osunsade, A., David, Y., Cesarman, E., Melnick, A. M., Sidoli, S., Garcia, B. A., Edelmann, W., Macian, F. \& Skoultchi, A. I. H1 histones control the epigenetic landscape by local chromatin compaction. Nature 1-6 (2020). doi:10.1038/s41586-020-3032-z 
39. Yusufova, N., Kloetgen, A., Teater, M., Osunsade, A., Camarillo, J. M., Chin, C. R., Doane, A. S., Venters, B. J., Portillo-Ledesma, S., Conway, J., Phillip, J. M., Elemento, O., Scott, D. W., Béguelin, W., Licht, J. D., Kelleher, N. L., Staudt, L. M., Skoultchi, A. I., Keogh, M.-C., Apostolou, E., Mason, C. E., Imielinski, M., Schlick, T., David, Y., Tsirigos, A., Allis, C. D., Soshnev, A. A., Cesarman, E. \& Melnick, A. M. Histone H1 loss drives lymphoma by disrupting 3D chromatin architecture. Nature 1-7 (2020). doi:10.1038/s41586-020-3017-y

40. Sollberger, G., Streeck, R., Apel, F., Caffrey, B. E., Skoultchi, A. I. \& Zychlinsky, A. Linker histone H1.2 and H1.4 affect the neutrophil lineage determination. Elife 9, e52563 (2020).

41. Mewes, H. W., Albermann, K., Bähr, M., Frishman, D., Gleissner, A., Hani, J., Heumann, K., Kleine, K., Maierl, A., Oliver, S. G., Pfeiffer, F. \& Zollner, A. Overview of the yeast genome. Nature 387, 7-8 (1997).

42. Kellis, M., Birren, B. W. \& Lander, E. S. Proof and evolutionary analysis of ancient genome duplication in the yeast Saccharomyces cerevisiae. Nature 428, 617-624 (2004). sequences that mediate targeting to the nucleus and nucleolus, and colocalization with human chromosomes. J Gen Virol 84, 3423--3428 (2003). One 10, e0137102 (2015).

45. Zhang, Y., Liu, T., Meyer, C. A., Eeckhoute, J., Johnson, D. S., Bernstein, B. E., Nusbaum, C., Myers, R. M., Brown, M., Li, W. \& Liu, X. S. Model-based Analysis of ChIP-Seq (MACS). Genome Biol 9, R137 (2008).

1148 46. Ishimi, Y. \& Kikuchi, A. Identification and molecular cloning of yeast homolog of nucleosome assembly protein I which facilitates nucleosome assembly in vitro. J Biological Chem 266, 7025-9 (1991).

1151 47. Lu, J., Kobayashi, R. \& Brill, S. J. Characterization of a High Mobility Group 1/2 Homolog 1152 in Yeast. J Biol Chem 271, 33678-33685 (1996). Biochemical and Phenotypic Characterization of Hho1p, the Putative Linker Histone H1 of 1155 Saccharomyces cerevisiae. J Biol Chem 13, (1998). transcription. Proc National Acad Sci 105, 14838-14843 (2008). 

Nap1 Promotes Nucleosome Assembly by Eliminating Nonnucleosomal Histone DNA Interactions. Mol Cell 37, 834-842 (2010).

51. Murugesapillai, D., McCauley, M. J., Huo, R., Holte, M. H. N., Stepanyants, A., Maher, L. J., Israeloff, N. E. \& Williams, M. C. DNA bridging and looping by HMO1 provides a mechanism for stabilizing nucleosome-free chromatin. Nucleic Acids Res 42, 8996-9004 (2014).

52. Herskowitz, I. Life cycle of the budding yeast Saccharomyces cerevisiae. Microbiol Rev 52, 536-53 (1988).

53. Muñoz-Barrera, M. \& Monje-Casas, F. The Mitotic Exit Network, Methods and Protocols. 35-44 (2016). doi:10.1007/978-1-4939-6502-1_3

54. Jonas, R. A., Ung, L., Rajaiya, J. \& Chodosh, J. Mystery Eye: Human Adenovirus and the Enigma of Epidemic Keratoconjunctivitis. Prog Retin Eye Res 76, 100826 (2019).

56. Vassilev, L. T., Tovar, C., Chen, S., Knezevic, D., Zhao, X., Sun, H., Heimbrook, D. C. \& Chen, L. Selective small-molecule inhibitor reveals critical mitotic functions of human CDK1. Proc National Acad Sci 103, 10660-10665 (2006).

57. Hendzel, M. J., Wei, Y., Mancini, M. A., Hooser, A. V., Ranalli, T., Brinkley, B. R., BazettJones, D. P. \& Allis, C. D. Mitosis-specific phosphorylation of histone H3 initiates primarily within pericentromeric heterochromatin during $\mathrm{G} 2$ and spreads in an ordered fashion coincident with mitotic chromosome condensation. Chromosoma 106, 348-360 (1997). phosphorylation is required for the initiation, but not maintenance, of mammalian chromosome condensation. J Cell Sci 111 ( Pt 23), 3497-506 (1998).

59. Crosio, C., Fimia, G. M., Loury, R., Kimura, M., Okano, Y., Zhou, H., Sen, S., Allis, C. D. \& Sassone-Corsi, P. Mitotic Phosphorylation of Histone H3: Spatio-Temporal Regulation by Mammalian Aurora Kinases. Mol Cell Biol 22, 874-885 (2002). Blanchard, K. \& Ye, X. S. Cdk1 Activity Is Required for Mitotic Activation of Aurora A during G2/M Transition of Human Cells. J Biol Chem 285, 21849-21857 (2010). single early family of $3^{\prime}$ coterminal mRNAs and five late families. Cell 22, 905-916 (1980). 
63. Ferrari, R., Berk, A. J. \& Kurdistani, S. K. Viral manipulation of the host epigenome for oncogenic transformation. Nat Rev Genet 10, 290-294 (2009). Transformed by DNA from Human Adenovirus Type 5. J Gen Virol 36, 59-72 (1977).

65. Zemke, N. R., Gou, D. \& Berk, A. J. Dedifferentiation by adenovirus E1A due to inactivation of Hippo pathway effectors YAP and TAZ. Gene Dev 33, 828-843 (2019).

66. Khandelia, P., Yap, K. \& Makeyev, E. V. Streamlined platform for short hairpin RNA interference and transgenesis in cultured mammalian cells. Proc National Acad Sci 108, 1279912804 (2011). DNA $\square$ adenovirus core protein VII and TAF $\square \mathrm{I} \beta / \mathrm{SET}$, an acidic molecular chaperone. Febs Lett 555, 521-527 (2003). 79, 2474-2483 (2005).

69. Kato, K., Okuwaki, M. \& Nagata, K. Role of Template Activating Factor-I as a chaperone in linker histone dynamics. J Cell Sci 124, 3254-3265 (2011). in chromatin. Biochimica Et Biophysica Acta Bba - Gene Regul Mech 1859, 462-467 (2016). W303, a Widely-Used Strain of Saccharomyces cerevisiae. G3 Genes Genomes Genetics 7, 2219-2226 (2017). Annu Rev Cell Dev Bi 34, 265-288 (2018). 
76. Irwin, N. A. T., Martin, B. J. E., Young, B. P., Browne, M. J. G., Flaus, A., Loewen, C. J. R., Keeling, P. J. \& Howe, L. J. Viral proteins as a potential driver of histone depletion in dinoflagellates. Nat Commun 9, 1535 (2018).

77. Shepard, R. N. \& Ornelles, D. A. E4orf3 is necessary for enhanced S-phase replication of cell cycle-restricted subgroup C adenoviruses. Journal of Virology 77, 8593-8595 (2003).

78. Turner, R. L., Groitl, P., Dobner, T. \& Ornelles, D. A. Adenovirus Replaces Mitotic Checkpoint Controls. J Virol 89, 5083-5096 (2015).

79. Turnell, A. S. \& Grand, R. J. DNA viruses and the cellular DNA-damage response. J Gen Virol 93, 2076-2097 (2012).

80. Wang, H., Ward, M. F., Fan, X.-G., Sama, A. E. \& Li, W. Potential Role of High Mobility Group Box 1 in Viral Infectious Diseases. Viral Immunol 19, 3-9 (2006).

81. Yang, H., Wang, H., Chavan, S. S. \& Andersson, U. High Mobility Group Box Protein 1 (HMGB1): The Prototypical Endogenous Danger Molecule. Mol Med 21, S6-S12 (2015).

82. Wei, J., Alfajaro, M. M., DeWeirdt, P. C., Hanna, R. E., Lu-Culligan, W. J., Cai, W. L., Strine, M. S., Zhang, S.-M., Graziano, V. R., Schmitz, C. O., Chen, J. S., Mankowski, M. C., Filler, R. B., Ravindra, N. G., Gasque, V., Miguel, F. J. de, Patil, A., Chen, H., Oguntuyo, K. Y., Abriola, L., Surovtseva, Y. V., Orchard, R. C., Lee, B., Lindenbach, B. D., Politi, K., Dijk, D. van, Kadoch, C., Simon, M. D., Yan, Q., Doench, J. G. \& Wilen, C. B. Genome-wide CRISPR screens reveal host factors critical for SARS-CoV-2 infection. Cell (2020). doi:10.1016/j.cell.2020.10.028

83. Bähler, J., Wu, J., Longtine, M. S., Shah, N. G., III, A. M., Steever, A. B., Wach, A., Philippsen, P. \& Pringle, J. R. Heterologous modules for efficient and versatile PCR $\square$ based gene targeting in Schizosaccharomyces pombe. Yeast 14, 943-951 (1998).

84. Longtine, M. S., III, A. M., Demarini, D. J., Shah, N. G., Wach, A., Brachat, A., Philippsen, P. \& Pringle, J. R. Additional modules for versatile and economical PCR $\square$ based gene deletion and modification in Saccharomyces cerevisiae. Yeast 14, 953-961 (1998).

85. Sikorski, R. S. \& Hieter, P. A system of shuttle vectors and yeast host strains designed for efficient manipulation of DNA in Saccharomyces cerevisiae. Genetics 122, 19-27 (1989).

86. Güldener, U., Heck, S., Fiedler, T., Beinhauer, J. \& Hegemann, J. H. A New Efficient Gene Disruption Cassette for Repeated Use in Budding Yeast. Nucleic Acids Res 24, 2519-2524 (1996).

87. Gietz, R. D. \& Woods, R. A. Transformation of yeast by lithium acetate/single-stranded carrier DNA/polyethylene glycol method. Methods Enzymol 350, 87-96 (2002).

88. Kushnirov, V. V. Rapid and reliable protein extraction from yeast. Yeast 16, 857-860 (2000). 
1259 89. Zentner, G. E., Tsukiyama, T. \& Henikoff, S. ISWI and CHD Chromatin Remodelers Bind 1260 Promoters but Act in Gene Bodies. Plos Genet 9, e1003317 (2013).

1261 90. Le, L. P., Le, H. N., Nelson, A. R., Matthews, D. A., Yamamoto, M. \& Curiel, D. T. Core 1262 labeling of adenovirus with EGFP. Virology 351, 291-302 (2006). 